\title{
Controlling the motion of interacting particles: Homogeneous systems and binary mixtures
}

\author{
Sergey Savel'eva) \\ Frontier Research System, The Institute of Physical and Chemical Research (RIKEN), Wako-shi, Saitama, \\ 351-0198, Japan \\ Franco Nori \\ Frontier Research System, The Institute of Physical and Chemical Research (RIKEN), Wako-shi, Saitama, \\ 351-0198, Japan and Center for Theoretical Physics, Department of Physics, Center for the Study \\ of Complex Systems, The University of Michigan, Ann Arbor, Michigan 48109-1120
}

(Received 3 January 2005; accepted 22 February 2005; published online 17 June 2005)

\begin{abstract}
We elaborate on recent results on the transport of interacting particles for both single-species and binary mixtures subject to an external driving on a ratchetlike asymmetric substrate. Moreover, we also briefly review motion control without any spatial asymmetric potential (i.e., no ratchet). Our results are obtained using an analytical approach based on a nonlinear Fokker-Planck equation as well as via numerical simulations. By increasing the particle density, the net dc ratchet current in our alternating (ac)-driven systems can either increase or decrease depending on the temperature, the drive amplitude, and the nature of the inter-particle interactions. This provides an effective control of particle motion by just changing the particle density. At low temperatures, attracting particles can condense at some potential minima, thus breaking the discrete translational symmetry of the substrate. Depending on the drive amplitude, an agglomeration or condensation results either in a drop to zero or in a saturation of the net particle velocity at densities above the condensation density - the latter case producing a very efficient rectification mechanism. For binary mixtures we find three ways of controlling the particle motion of one (passive) $B$ species by means of another (active) $A$ species: (i) Dragging the target particles $B$ by driving the auxiliary particles $A$, (ii) rectifying the motion of the $B$ particles on the asymmetric potential created by the $A-B$ interactions, and (iii) dynamically modifying (pulsating) this potential by controlling the motion of the $A$ particles. This allows to easily control the magnitude and direction of the velocity of the target particles by changing either the frequency, phase and/or amplitude of the applied ac drive(s). () 2005 American Institute of Physics. [DOI: 10.1063/1.1903183]
\end{abstract}

Stochastic transport on periodic asymmetric substrates far from equilibrium has raised wide-spread interest in the recent literature. ${ }^{1}$ Various realizations of ratchet systems working out of equilibrium have been proposed involving different rectification mechanisms, like timedependent temperature oscillations (temperature ratchet ${ }^{2}$ ), zero-average sinusoidal alternating (ac) forces (ac tilted or rocked ratchet ${ }^{3}$ ), stochastic and deterministic fluctuations of the ratchet potentials, ${ }^{4}$ among others. Intense research activity in this field is partly motivated by the challenge to describe and control some biological processes at both the cell level (for instance, transport in ion channels ${ }^{5}$ ) and the body level (muscle operations ${ }^{6}$ ). Moreover, recent technological advances have allowed the development of devices to guide tiny particles on nano- and micro-scales, ${ }^{7}$ e.g., for particle separation techniques, ${ }_{9}^{8}$ smoothing of atomic surfaces during electromigration, and superconducting vortex motion control. ${ }^{10,11}$ Some of these devices have been realized experimentally to manipulate the motion of vortices in superconductors, ${ }^{12-15}$ particles in asymmetric silicon pores ${ }^{16}$ as well as charged particles through artificial pores ${ }^{17}$ and arrays of optical

${ }^{a)}$ Electronic mail: ssavelev@ riken.jp tweezers, ${ }^{18-22}$ among others. However, the interaction among particles is expected to have an important role on the rectification power of ratchets. Indeed, it has been found that interactions can result in very unusual transport properties, ${ }^{10,23-30}$ including spontaneous symmetry breaking, commensurability effects, unusual negative mobility, and surprising current inversions.

\section{INTRODUCTION}

We show that the net current can be effectively controlled by changing the density of interacting particles. This dependence can be described using effective potentials ${ }^{31}$ which take into account the renormalization of the bare substrate potential $U$ due to particle interactions. For repelling particles, $U$ flattens with increasing particle density because particles repel one another from the potential wells. This results in an increase of the net current for lower (with respect to the potential barriers) drives and a decrease for stronger drives.

The effective potential becomes deeper with increasing particle density of attractive particles resulting in the opposite behavior with respect to the repulsive particles. ${ }^{31,32}$ 
Moreover, the agglomeration or "condensation",32 of attractive particles into the substrate potential wells occur at large enough particle densities. This results in the sudden drop of their drift velocity at the condensation point for low drives. On raising the drive amplitude it can happen that the particles pile-up or condense only when the ac force pushes them against the steeper slope of the asymmetric substrate wells, while remaining in the running state as they are driven in the opposite direction. For such a range of large drive amplitudes, the current, versus particle density, saturates at a maximal value in the vicinity of the condensation density. ${ }^{32}$

Recent experiments on transport of $\mathrm{K}$ and $\mathrm{Rb}$ ions in an ion channel, ${ }^{33}$ particles of different size in asymmetric silicon pores, ${ }^{16}$ pinned and interstitial vortices, ${ }^{12}$ and two different types of vortices ${ }^{13,14}$ pose the question of how directed motion of two or more types or species of particles affect one another. More interestingly, one might wonder how to induce and control the net transport of passive particles, which are insensitive to the applied drives and/or substrates. A way to tackle this challenging problem is to employ auxiliary $A$ particles that: (i) Interact with the target species (the $B$ particles) and (ii) are easy to drive by means of external forces. By driving $A$ particles one can regulate the motion of otherwise passive $B$ particles through experimentally accessible systems, like ion channels, ${ }^{33}$ artificial pores, ${ }^{16,17}$ arrays of optical tweezers, ${ }^{18}$ or certain superconducting devices ${ }^{10-14}$ ).

In order to study the influence of the interspecies interaction on particle transport in a binary mixture, we consider external forces applied either to the $A$ particles only or simultaneously to both the $A$ and $B$ types of particles. We have found three ways to control particles in binary mixtures: ${ }^{11,31,32,34,35}$

1. Dragging $B$ by $A$ : When a driving force acts on only one component (e.g., $A$ ) of a binary ( $A$ and $B$ particles) mixture and there is no substrate at all, the driven species can drag along the passive particles. The ac dragging effect can be described as follows: if the driving force $f_{A}(t)$ acting on the active species $A$ is zero-averaged but asymmetric in time, a net motion of both species occurs. This enables control of the motion of both $A$ and $B$ particle species, even when there is no asymmetric substrate (or no substrate at all).

2. Mediated ratchet effect: If only one species feels an asymmetric substrate, then these "active" $A$ particles produce an effective asymmetric potential for the other component $B$ (due to interspecies interaction). This potential has the same (opposite) polarity for attractive (repulsive) interaction between different species and can be used for the rectification of either the ac or random motion of passive particles.

3. Gating effect (flashing effective potentials): The motion of active particles in the "hard" direction with respect to the asymmetric substrate produces high potential barriers for the passive particles, stopping the motion of the passive species. The time-correlation of the stopping intervals and ac-driving of passive particles creates another way to control the motion in the binary mixture. Changing the relative phases of the driving forces makes it possible to guide at will both the $A$ and $B$ species either in the same or in opposite directions.

These three ways for manipulating tiny particles in mixtures allow an unprecedented level of motion control of different components by tuning the phase and/or amplitude and/or frequency of the applied drives.

Systems where our two-species transport technique might be implemented with success are the focus of ongoing experimental work (e.g., superconducting samples penetrated by topologically different vortices, ${ }^{11,13,14}$ ion channel traversed by competing ion species ${ }^{33}$ ).

\section{ONE TYPE OF PARTICLE}

\section{A. Langevin and Fokker-Planck approaches}

Our starting point for a system of identical particles (i.e., with only one species) is the set of Langevin equations

$$
\dot{x}_{i}=-\frac{\partial U\left(x_{i}\right)}{\partial x_{i}}-\sum_{j \neq i} \frac{\partial}{\partial x_{i}} W\left(x_{i}-x_{j}\right)+F(t)+\sqrt{2 k_{B} T} \xi^{(i)}(t),
$$

for interacting particles moving on the one-dimensional asymmetric periodic potential $U, U(x+l)=U(x)$, in the presence of a time-periodic force $F(t)$ with frequency $\nu$. Here, the Gaussian white noise $\xi^{(i)}(t)$ with zero average $\left\langle\xi^{(i)}\right\rangle=0$ satisfies the fluctuation-dissipation relation $\left\langle\xi^{(i)}(t) \xi^{(i)}(t+\tau)\right\rangle$ $=\delta(\tau)$; where $T$ is the temperature, $k_{B}$ is the Boltzmann constant, and $W$ denotes the pair interaction potential. Indices $i$ and $j$ run over all particles. For simplicity, we set the viscous coefficient equal to one (Smoluchowski approximation). We integrated the set of equations (1) for our numerical simulations, while the analytical predictions reported below were derived by solving the integro-differential equations for the corresponding many-particle distribution functions. The Fokker-Planck-type equation for the one-particle distribution function, $F_{1}(t, x)$, can be written in the form: ${ }^{31,32}$

$$
\begin{aligned}
\frac{\partial F_{1}(t, x)}{\partial t}= & \frac{\partial}{\partial x}\left[\left(\frac{\partial U(x)}{\partial x}-F(t)\right) F_{1}(t, x)\right] \\
& +\frac{\partial}{\partial x} F_{1}(t, x) \int d \widetilde{x} F_{1}(t, \tilde{x}) G(t, x, \tilde{x}) \frac{\partial W(x-\tilde{x})}{\partial x} \\
& +k_{B} T \frac{\partial^{2} F_{1}(t, x)}{\partial x^{2}}
\end{aligned}
$$

where

$$
F_{2}(t, x, \tilde{x}) \equiv F_{1}(t, x) F_{1}(t, \tilde{x}) G(x, \tilde{x}, t),
$$

denotes a binary distribution function. It is apparent that particle-particle correlations decay on a scale of the order of either the interaction length $\lambda$ for low particle densities ( $n$ $\ll 1 / \lambda$ ) or the inter-particle distance $1 / n$ for high particle densities $(n \gg 1 / \lambda)$. As a consequence, the function $G$, which describes the particle-particle correlation, differs appreciably from 1 (uncorrelated particle motion) for particle separations $|x-\tilde{x}| \lesssim \min \left\{n^{-1}, \lambda\right\}$, only. This has been numerically proven in Ref. 31 and is shown in Fig. 1(a). Therefore, if each particle interacts with many neighbors, i.e., $n \lambda \gg 1$, the function 

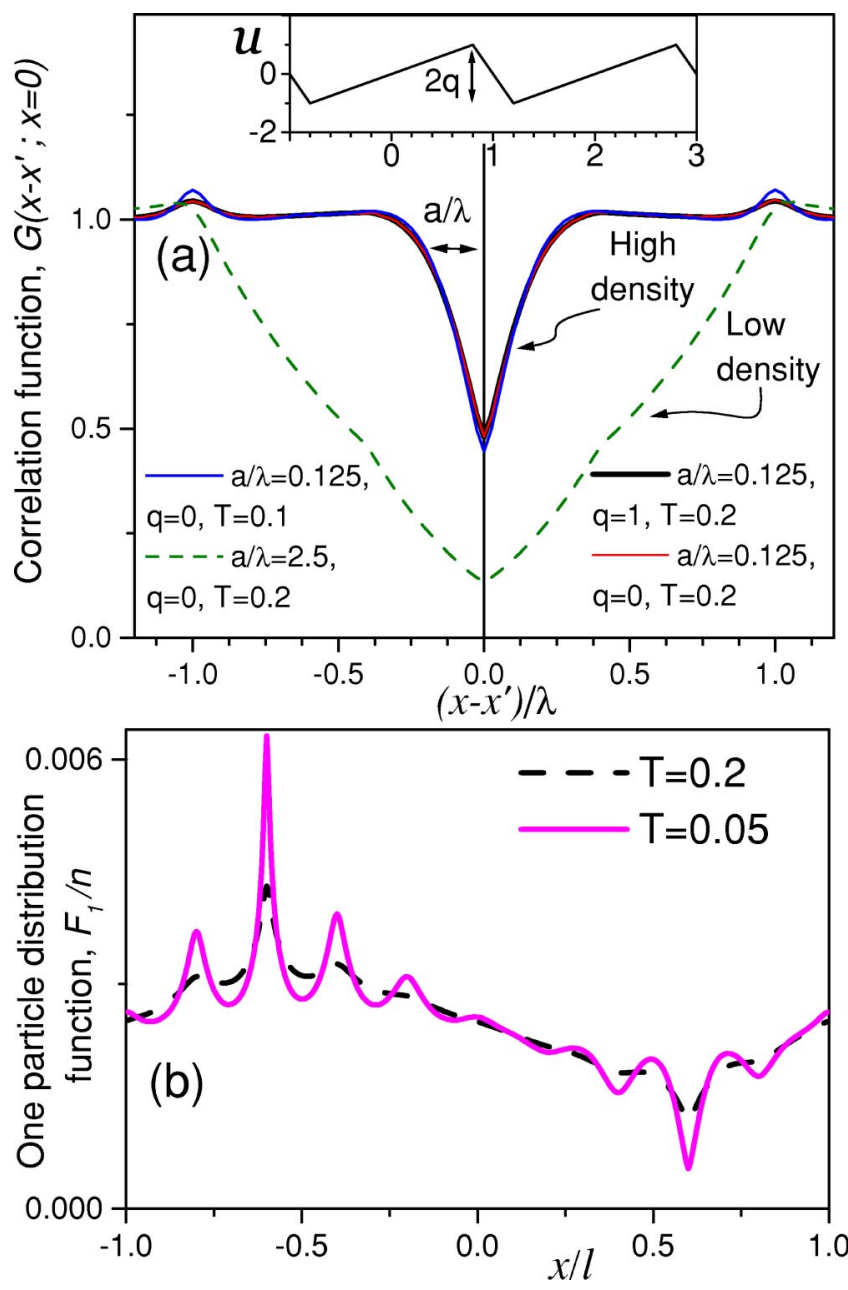

FIG. 1. (a) Correlation function $G$ expressing the deviation $(G-1)$ from the mean-field approximation (the mean-field approach assumes $G=1$ ) vs distance $x-x^{\prime}$ between particles, normalized by the interaction length $\lambda$. The interaction was taken as $W(x)=g(\lambda-|x|) / \lambda^{2}$, while the substrate potential $U(x / l)$ is shown in the inset. $G$ substantially differs from one over distance $\left|x-x^{\prime}\right| \sim a$, when the average distance $a$ between particles is much smaller than $\lambda$. In the opposite limit, $a>\lambda$, we obtain $G<1$, on scales $\left|x-x^{\prime}\right|<\lambda$. This result depends neither on temperature nor the substrate potential $U$ in the studied range of parameters. (b) Oscillations of the normalized oneparticle distribution function $F_{1}(x / l) / n$ due to nonlocal effects for the potential shown in the inset of (a), $Q=1, l_{2} / l_{1}=2 / 3, l=l_{1}+l_{2}=2 ; \lambda=0.2$. A large density of particles in the bottom of potential wells $x=x_{\text {min }}$ repels other particles from the nearby region of a scale of about $\lambda$, effectively shifting the new energetically favorable positions to $x_{\min } \pm \lambda$ and so on-producing oscillations in $F_{1}(x)$. These results were obtained by numerically solving Eq. (1).

$G$ in Eq. (2) can be safely approximated to 1 over the entire integration domain (of order $\lambda$ ) of $\int d \widetilde{x} F_{1} G \partial W / \partial x$. It follows that Eq. (2) can be reduced to its mean-field (MF) form $^{31,32,34,35}$

$$
\begin{aligned}
\frac{\partial F_{1}(t, x)}{\partial t} & =-\frac{\partial j}{\partial x} \\
& =\frac{\partial}{\partial x}\left[F_{1}(t, x)\left\{\frac{\partial U^{\mathrm{MF}}}{\partial x}-F(t)\right\}+k_{B} T \frac{\partial F_{1}(t, x)}{\partial x}\right] .
\end{aligned}
$$

where the mean-field potential $U^{\mathrm{MF}}(x)$ is defined as

$$
U^{\mathrm{MF}}(x)=U(x)+\int d x^{\prime} W\left(x-x^{\prime}\right) F_{1}\left(t, x^{\prime}\right)
$$

Hereafter, the one-particle distribution function $F_{1}(t, x)$ is normalized in terms of the average particle density $n$, i.e.,

$$
\int_{0}^{l} F_{1}(t, x) d x / l=n .
$$

If the spatial scale of the substrate potential is of the order of the interaction length $\lambda$, the nonlocality can play an important role, producing, e.g., oscillations of the density $F_{1}(x)$ [see Fig. 1(b)]. In this case, the analytical treatment of the integro-differential equation (4) is very complicated.

In order to make the problem more tractable, we further discard nonlocal effects by assuming the interaction length $\lambda$ to be much smaller than the period $l$ of the substrate potential $U(x)$. This allows us to replace the integro-differential equation (4) by

$$
\begin{aligned}
\frac{\partial F_{1}(t, x)}{\partial t}= & \frac{\partial}{\partial x}\left[\left(\frac{\partial U(x)}{\partial x}-F(t)\right) F_{1}(t, x)+g F_{1} \frac{\partial F_{1}}{\partial x}\right] \\
& +k_{B} T \frac{\partial^{2} F_{1}(t, x)}{\partial x^{2}}
\end{aligned}
$$

with

$$
g \equiv \int_{-\infty}^{\infty} d x^{\prime} W\left(x-x^{\prime}\right)=\int_{-\infty}^{\infty} d y W(y) .
$$

Therefore, Eq. (7) is valid under the following restrictions:

$$
n^{-1} \ll \lambda \ll l .
$$

Note that, even though we assumed locality with respect to the substrate unit length, the interparticle interaction can still be regarded as a long-range interaction because of the density requirement $\lambda \gg 1 / n$. These are the approximations under which in the following sections we solve Eq. (7) analytically and compare our analytical results with data from numerical simulations based on the Langevin equations (1). Although conditions (9) strictly apply to a somewhat limited class of physical systems, the results obtained below have much wider applicability. Indeed, numerical simulations performed well outside the parameter region (9) agree quite closely with our mean-field description.

\section{B. Close to equilibrium: Effective potentials and effective temperature}

The starting point of our analysis is the derived nonlinear Fokker-Planck equation for a rocked ratchet

$$
\frac{\partial F_{1}}{\partial t}=\frac{\partial}{\partial x}\left(F_{1}\left(\frac{d U}{d x}-F(t)\right)+g F_{1} \frac{\partial F_{1}}{\partial x}+k_{B} T \frac{\partial F_{1}}{\partial x}\right) .
$$

A periodic square-wave force keeps the system out of equilibrium

$$
F(t)=A \operatorname{sgn}[\cos (\omega \mathrm{t})],
$$

with $\operatorname{sgn}[\cdots]$ denoting the sign of the argument and $\omega=2 \pi \nu$ the driving frequency. 
In the general case, an analytical study of Eq. (10) is too complicated. However, if the period of the force oscillations $2 \pi / \omega$ is much shorter than the other characteristic time scales in the problem, or $F(t)$ is rather weak, it is reasonable to expect that the system relaxes close to the equilibrium state corresponding to zero applied force. This equilibrium solution

$$
F_{1}(x, A=0) \equiv \phi_{0}=\phi_{0}(x)
$$

satisfies the nonlinear equation

$$
U^{\prime}(x) \phi_{0}+g \phi_{0} \frac{d \phi_{0}}{d x}+k_{B} T \frac{d \phi_{0}}{d x}=0,
$$

which can be solved in implicit form,

$$
C(n) \exp \left(-\frac{U(x)}{k_{B} T}\right)=\phi_{0}(x) \exp \left(\frac{g}{k_{B} T} \phi_{0}(x)\right) \equiv Z\left(\phi_{0}\right)
$$

where the constant $C(n)$ is determined by the normalization condition

$$
\int_{0}^{l} d x \phi_{0}(x)=n l .
$$

The equilibrium distribution $\phi_{0}$ coincides with the usual Boltzmann distribution if the particle interaction is switched off, $g=0$.

The equation for the perturbation correction $\psi=\psi(x)$ $\equiv F_{1}-\phi_{0}$ from the equilibrium state $\phi_{0}$ is

$$
\begin{aligned}
\frac{\partial \psi}{\partial t}= & \frac{\partial}{\partial x}\left(\psi\left(\frac{d U^{\mathrm{eff}}}{d x}-F(t)\right)+k_{B} T^{\mathrm{eff}} \frac{\partial \psi}{\partial x}+g \psi \frac{\partial \psi}{\partial x}\right. \\
& \left.-F(t) \phi_{0}\right),
\end{aligned}
$$

while the effective potential and temperature are defined as

$$
\begin{aligned}
& U^{\mathrm{eff}}(x)=U(x)+g \phi_{0}, \\
& k_{B} T^{\mathrm{eff}}(x)=k_{B} T+g \phi_{0} .
\end{aligned}
$$

Equation (15) can be solved perturbatively (see, e.g., Ref. 31). This perturbation approach can be qualitatively interpreted if we separate the running particles, a relatively small fraction of about $|\psi(x)| / \phi_{0}(x)$ at the point $x$, from those trapped at the substrate minima. The moving particles feel the potential $U^{\text {eff }}$ generated by both the substrate and the trapped particles.

In the case of repulsive particle interaction, such an effective potential is smoother than the bare substrate potential [see Fig. 2(b)] because the particles occupying the bottom of the potential wells tend to repel the running particles away from the potential minima.

In contrast to this, when increasing the density of attracting particles, the wells of the effective potential grow even deeper than the substrate wells [Fig. 3(b)]. Note that the particle-particle interaction also induces a spatial dependence of the effective temperature [Figs. 2(a) and 3(a)], which implies a spatial dependence of the diffusion constant of the running particles. The effective temperature and potential exhibit the same asymmetry (polarity) for the case of
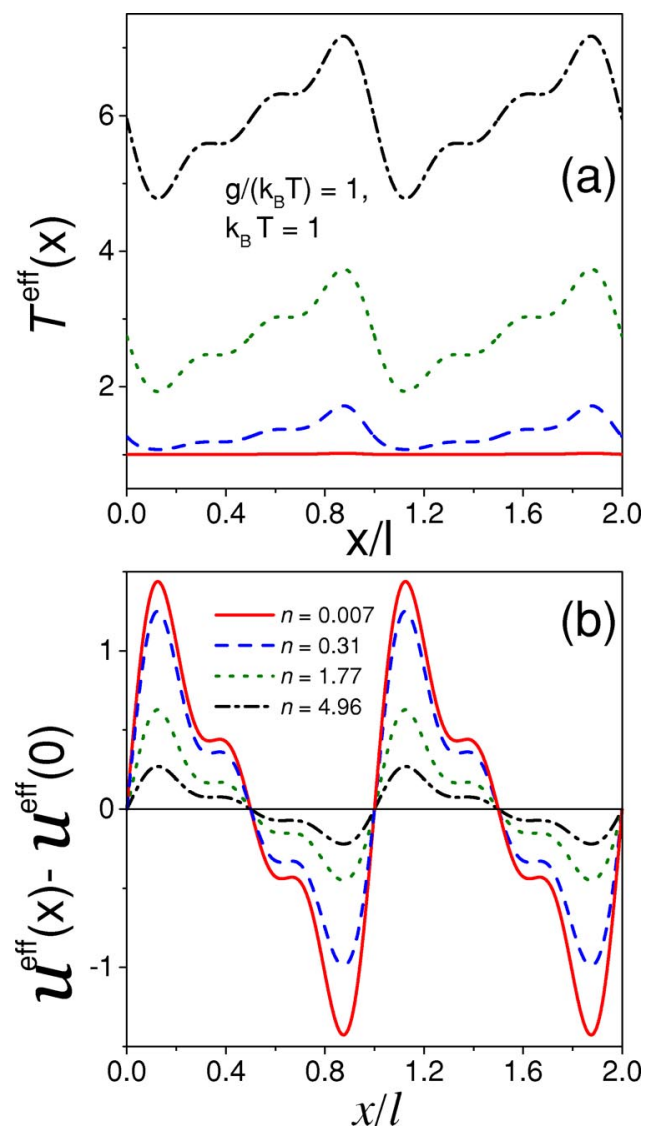

FIG. 2. (a) The spatial dependence of the effective temperature $T^{\mathrm{eff}}$, and (b) the effective potential $U^{\text {eff }}-U^{\text {eff }}(0)$ for repelling particles and for different values of their density $n$. Both the effective temperature $T^{\mathrm{eff}}$ and the effective potential energy $U^{\text {eff }}$ are shown in arbitrary units. The bare substrate potential is chosen as: $U(x)=U^{\operatorname{ramp}}(x) \equiv \sin (2 \pi x / l)+\frac{1}{2} \sin (4 \pi x / l)$ $+\frac{1}{3} \sin (6 \pi x / l)$. The mutual repulsion of particles causes the flattening of the "effective" potential with increasing $n$. The positions of the maxima of $U^{\text {eff }}(x)$ coincide with the minima of $T^{\mathrm{eff}}(x)$, and vice versa. This indicates that $T^{\mathrm{eff}}(x)$ and $U^{\mathrm{eff}}(x)$ have opposite asymmetry.

attracting particles [Fig. 3(a)], meaning that the positions of their maxima and minima coincide. In this respect, we say that for repelling particles, the effective temperature and the effective potential have opposite asymmetry [Fig. 2(a)].

Equation (14) always admits a solution if the particles repel each other, $g>0$. However, in the case of attracting particles, $g<0$, the transcendental Eq. (14) has a solution only if

$$
C(n) \exp \left(-\frac{\min (U(x))}{k_{B} T}\right)<\frac{k_{B} T}{e|g|} .
$$

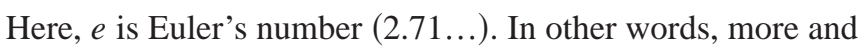
more particles accumulate near $x_{\min }$, which in turn attract additional particles from even further away. Eventually, the particle attraction wins over the random thermal noise. This occurs at a critical value $n_{\text {crit }}$ of the particle density when $Z\left(\phi_{0}\left(x_{\min }\right)\right)$ equals the maximum value $\max _{\phi_{0}}\left[Z\left(\phi_{0}\right)\right]$ $=k_{B} T / e|g|$. At higher densities, the equilibrium distribution (14) cannot be sustained any longer; thermal noise cannot prevent the condensation of a finite fraction of the nonideal (interacting) gas particles into the liquidlike phase at the bot- 

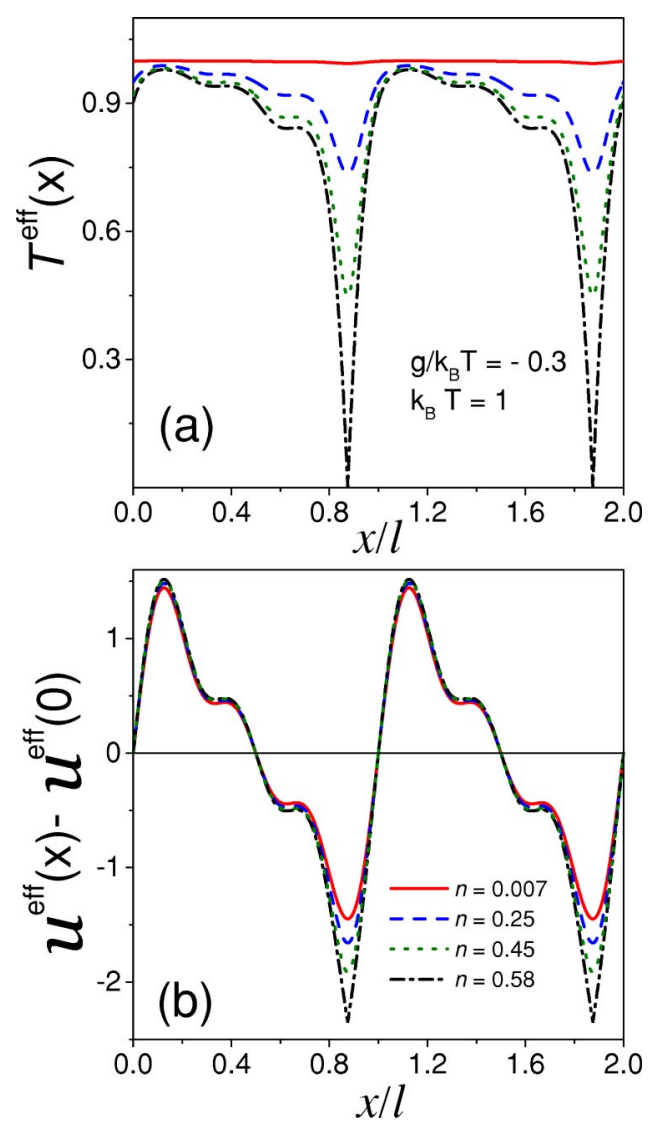

FIG. 3. Same as in Fig. 2, but for attracting particles. The "effective" potential wells deepen due to particle accumulating near the potential minima. The effective temperature $T^{\text {eff }}$ has a maximum where $U^{\text {eff }}$ has a maximum, and vice versa. The sudden agglomeration or condensation of the attracting particles in the potential minima $x=x_{\min }$ occurs when $T^{\mathrm{eff}}\left(x_{\min }\right)$ drops to zero.

tom of the potential wells. This results in a drop of effective diffusion coefficient

$$
D^{\text {eff }} \propto T^{\text {eff }}
$$

to zero [see upper panel in Fig. 3(a)] and the mobility of the particles also becomes zero.

\section{Far from equilibrium: Rectification enhancement and particle condensation}

In order to understand how to control particle motion by changing the particle density, we recall that the effective potential acting on moving particles flattens when increasing the density of the repelling particles.

This mechanism is illustrated schematically in Fig. 4(a) where a typical effective potential at low (solid red curve) and high density (dotted blue curve) is drawn for clarity. If the temperature and the amplitude of the ac force are low enough, a running particle cannot overcome the potential barriers for low particle density [solid red curve, Fig. 4(b)]. Therefore, the particle (solid circle) remains trapped in a potential minimum during the ac tilting of the potential [in Fig. 4(b), the upper and bottom panels show the effective potential subject to maximum tilt both to the right and to the left]. Thus, the current has to be very small.

However, the particle can overcome the lower potential
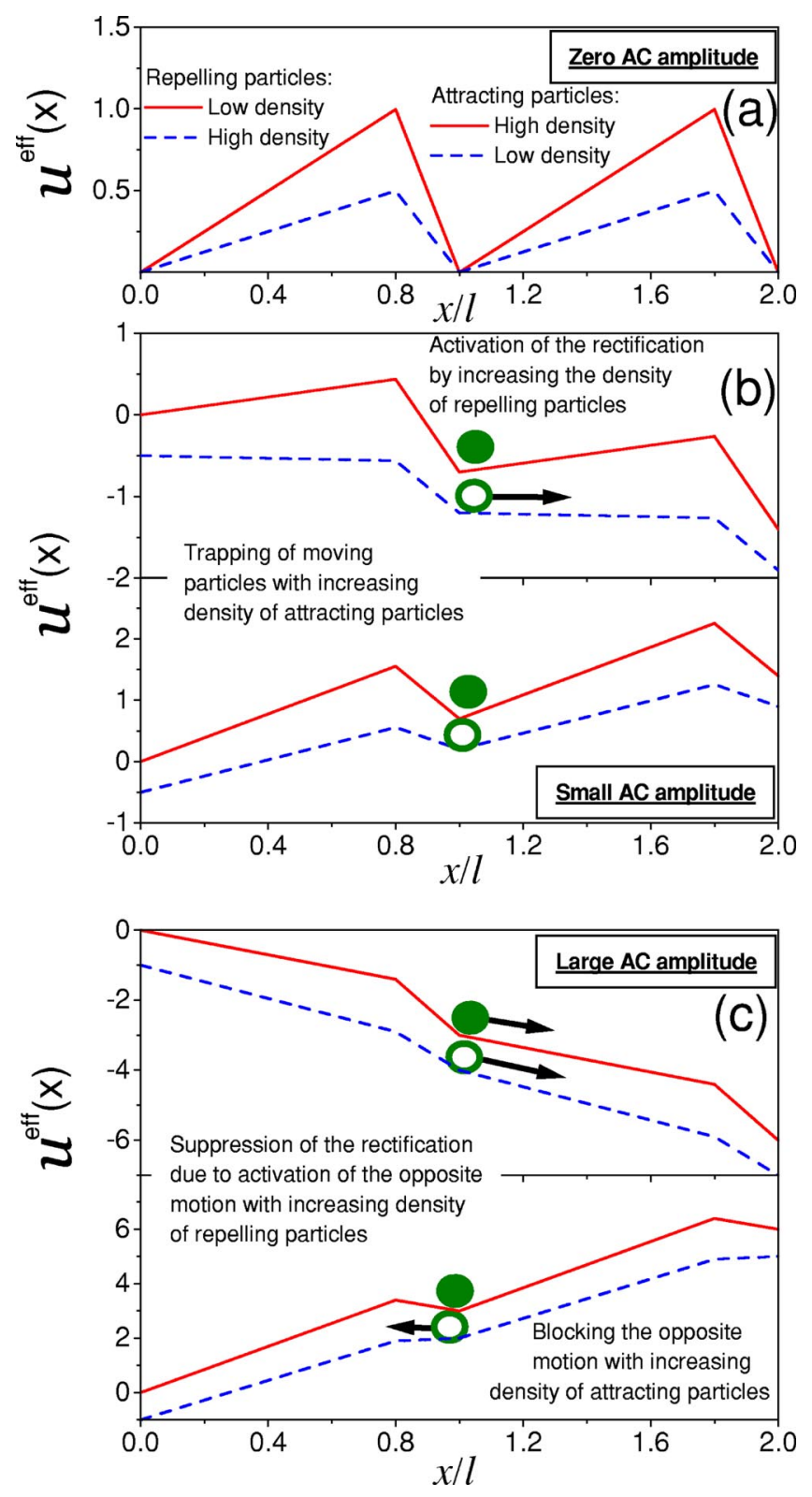

FIG. 4. Schematics of the density-dependence of the net time-average velocity $V_{\text {dc }}$ for a rocked ratchet: (a) The original potential (solid red line) and flatter effective potential (dashed blue line) $U^{\text {eff }}(x)$ due to the repelling interaction among particles; (b) The small amplitude of the ac force, which tilts the effective potential from $U^{\text {eff }}-A x$ (the upper panel) to $U^{\text {eff }}+A x$ (bottom panel), could not produce a net motion in the bare potential (solid red line) at low temperatures because of the potential barriers. However, the suppressed barriers (dashed blue line) for a high density of repelling particles can be overcome, resulting in directed particle motion; (c) For large amplitudes, the ac particle motion in the bare potential gets rectified as the tilt is strong enough. Indeed, a particle (solid circle) moves easily only when the potential is tilted to $U^{\text {eff }}-A x$ [upper panel in (c)]; the suppression of the barriers also activates a substantial particle flow in the opposite direction, thus reducing the ratchet rectification power. For attracting particles, the effective potential $U^{\text {eff }}(x)$ deepens with increasing the density $n$. The dependence of the net average velocity $V_{\mathrm{dc}}$ on the density $n$ for attracting particles is discussed in the text.

barriers of the effective potential corresponding to higher particle density $n$ (dotted blue curve), when the potential is tilted [Fig. 4(b), a particle (open circle) can move to the left]. The above behavior leads to the "activation" of the net motion for higher $n$ due to flattening effective potential with 
increasing $n$ (schematically shown as a transformation of $U$ from the solid red curve to dashed blue one). Thus, the dc net current is obviously enhanced when increasing the density of particles for small enough amplitude $A$ of the ac force.

On the other hand, if $A$ is strong enough, particles can easily pass through the potential barriers in the preferable direction even for low particle density when the potential is tilted [Fig. 4(c), upper panel, solid red curve]. In such a case, a particle (solid circle) moves easily to the left, while barriers prevent the motion in the opposite direction [Fig. 4(c), bottom panel, solid red curve], resulting in an effective rectification. The suppression of the barriers (associated with increasing the density $n$ of repelling particles) stimulates the undesirable motion in the direction which is opposite to the net current [Fig. 4(c), bottom panel, dotted blue curve]. With increasing $A$, this has to result in a change of the dependence of the net velocity $V_{\mathrm{dc}}$ on the particle density $n$, i.e., from an increasing to a decreasing function of $n$. This change of the $V_{\text {dc }}(n)$-dependence was observed in both simulations and analytical calculations [Fig. 5(a)].

For attracting particles, the potential wells deepen with increasing particle density. In Fig. 4 this corresponds to the modification of the effective potential $U^{\text {eff }}$ from a dashed blue (low density) to a solid red (high density) profile. Thus, particles (open circle), moving on the bare potential (low density case), get trapped by the deeper potential wells at higher densities [Fig. 4(b), solid circle, solid red curve]; at small $A$, the net velocity diminishes in the case of attracting particles. For higher driving amplitudes, the deepening effective potential, when increasing $n$, results in increasing ratchet asymmetry and, thus, increasing the net velocity. This is consistent with the results displayed in Fig. 5(b), green solid squares; inset in Fig. 5.

Moreover, for the case of attractive particles, there is another way to control particle motion, including strong enhancement of rectification power. Indeed, all our arguments on the basis of an effective potential $U^{\text {eff }}(x)$ become invalid for particle densities $n$ higher than $n_{\text {crit }}$, where a drivedependent condensation transition occurs. For rather small driving forces $F(t)$, this results in the sudden drop of their drift velocity at the condensation (agglomeration) point [Fig. 5(b), red open squares]. When raising the drive amplitude it can happen that the particles condense only when the ac force pushes them against the steeper slope of the asymmetric substrate wells, while remaining in the running state as they are driven in the opposite direction [Fig. 5(c), inset]. For such a range of large drive amplitudes, the ratchet current versus the particle density saturates at a maximal value in the vicinity of the condensation density [Fig. 5(c), solid red squares].

\section{Adiabatic approximation: Quantitative description far from equilibrium}

In the low frequency limit, for any time $\widetilde{t}$, the system can be regarded as being in the steady state corresponding to an applied dc force $F \equiv F(\widetilde{t})$; hence, the adiabatic expression for the ratchet current is $j_{\mathrm{dc}}=\nu \int_{0}^{1 / \nu} j[F(\widetilde{t})] d \widetilde{t}$, where $j(F)$ is the stationary current in the presence of the constant drive $F$. If $j(F)$ is not an odd function of $F[$ i.e., $j(F) \neq-j(-F)]$, then the
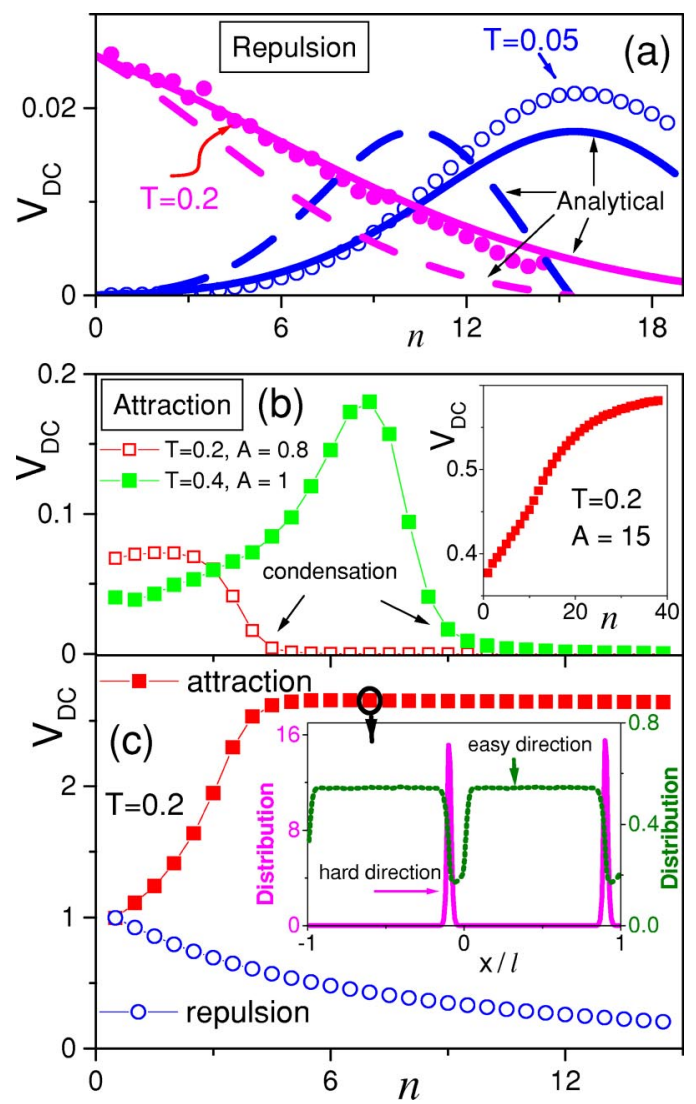

FIG. 5. Numerical data from Eqs. (1) with periodic boundary conditions over two potential unit cells: (a) time-average net velocity $V_{\mathrm{dc}}$ vs repelling particle density $n$ for $\nu=0.01, A=0.5, Q=1, l_{1}=0.9, \lambda=0.1, g_{\mathrm{MD}}=0.02, T$ $=0.2$ (solid magenta circles), and $T=0.05$ (open blue circles). The corresponding analytical predictions from Eqs. (22) for $g=g_{\mathrm{MD}}=0.02$ (dashed lines) and $g=g_{\mathrm{MF}}=0.02 / 1.5 \approx 0.0133$ (solid line) are reported for comparison (see text); (b) $V_{\mathrm{dc}}$ versus attracting particle density $n$ for $A=0.8$ and $T$ $=0.2$ (red open squares), $A=1$ and $T=0.4$ (green solid squares), $A=15$ and $T=0.2$ (inset); other parameter values are $\nu=0.01, Q=1, l_{1}=0.9, \lambda=0.1$, and $g=-0.02$; (c) $V_{\text {dc }}$ vs repelling (blue open circles) and attracting (red solid squares) particle density $n$ for $\nu=0.01, A=6, Q=1, l_{1}=0.9, \lambda=0.1, g=$ -0.02 , and $T=0.2$. Inset: Spatial distribution of attracting particles with $n$ $=7$ and other parameters as in the main panel (black circle). One snapshot (distribution $\equiv F_{1} / n$ ) of the particles was taken at each drive period with the external force pushing in the "hard" (magenta solid line, the left axis) or the "easy" (green dotted line, the right axis) direction, respectively.

rocked ratchet can rectify the oscillatory motion of the particles. The stationary solution to Eq. (4) can be written as

$$
-j(F)=\left(U^{\prime}-F\right) F_{1}(x)+k_{B} T F_{1}^{\prime}+g F_{1} F_{1}^{\prime},
$$

(' denoting an $x$ derivative). When adopting, for simplicity, the piecewise linear periodic potential $U(x+l)=U(x)$ :

$$
U(x)=Q \frac{x}{l_{1}} \quad \text { for } 0<x<l_{1}
$$

and

$$
U=Q-Q \frac{x-l_{1}}{l_{2}} \text { for } l_{1}<x<l_{1}+l_{2}=l,
$$

the stationary one-particle distribution in Eq. (18), $F_{1}(x)$, can be expressed in implicit form as 


$$
\frac{F_{1}\left(F-f_{1}\right)-j}{P_{0}\left(F-f_{1}\right)-j}=\exp \left(\frac{\left(F-f_{1}\right) x-g\left(F_{1}-P_{0}\right)}{k_{B} T+g j /\left(F-f_{1}\right)}\right),
$$

for $0<x<l_{1}$, and

$$
\frac{F_{1}\left(F+f_{2}\right)-j}{P_{1}\left(F+f_{2}\right)-j}=\exp \left(\frac{\left(F+f_{2}\right)\left(x-l_{1}\right)-g\left(F_{1}-P_{1}\right)}{k_{B} T+g j /\left(F+f_{2}\right)}\right),
$$

for $l_{1}<x<l$. Here, $P_{0}$ and $P_{1}$ are the particle densities at the potential minima and maxima, respectively, i.e., $P_{0}=F_{1}(0)$, $P_{1}=F_{1}\left(l_{1}\right) ; f_{1}=Q / l_{1}$, and $f_{2}=Q / l_{2}$ are the two restoring forces exerted by the substrate.

Two equations for the three unknown quantities $P_{0}, P_{1}$, and $j$ were derived writing Eqs. (20) and (21) for the extremal points $x=l_{1}$ and $x=l$, respectively, and imposing periodic boundary conditions $F_{1}(l)=F_{1}(0)=P_{0}$. A third equation for these variables was obtained by integrating Eq. (18) over one unit cell of the piecewise linear potential $U(x)$ and then eliminating the two integration constants $\int_{0}^{l_{1}} F_{1}(x) d x$ and $\int_{l_{1}}^{l} F_{1}(x) d x$ by means of the normalization condition $\int_{0}^{l} F_{1}(x) d x=n l$. The resulting equations can be conveniently expressed as

$$
\begin{aligned}
& \mathcal{P}-\frac{V}{f^{-}}=-\frac{\Delta}{2} \cot \left\{\frac{f^{-} / \Gamma_{1}+\widetilde{g} \Delta}{2\left[1+\widetilde{g} V / f^{-}\right]}\right\}, \\
& \mathcal{P}-\frac{V}{f^{+}}=\frac{\Delta}{2} \cot \left\{\frac{f^{+} / \Gamma_{2}-\widetilde{g} \Delta}{2\left[1+\widetilde{g} V / f^{+}\right]}\right\}, \\
& V=\frac{f^{-} f^{+}+(\widetilde{g} \mathcal{P}+1) \Delta q \Gamma_{1} \Gamma_{2}}{f+q\left(\Gamma_{2}-\Gamma_{1}\right)},
\end{aligned}
$$

in terms of the dimensionless variables $\mathcal{P}=\left(P_{0}+P_{1}\right) / 2 n, \Delta$ $=\left(P_{0}-P_{1}\right) / n, V=j l / k_{B} T n$, and the model parameters $q$ $=Q / k_{B} T$ (activation), $f=F l / k_{B} T$ (drive), $\tilde{g}=g n / k_{B} T$ (density or pair coupling), and $\gamma=l_{1} / l_{2}$ (ratchet anisotropy). Here, we introduce the auxiliary anisotropy parameters $\Gamma_{1}=l / l_{1}=1$ $+\gamma^{-1}$ and $\Gamma_{2}=l / l_{2}=1+\gamma$, as well as the total dimensionless forces $f^{-}=f-q \Gamma_{1}$ and $f^{+}=f+q \Gamma_{2}$ experienced by a single particle moving along the relevant sides of a potential well.

At low densities the net particle velocity $V$ can be expanded in powers of $\tilde{g}, V=V_{0}+\widetilde{g} V_{1}+\mathcal{O}\left(\widetilde{g}^{2}\right)$, where $V_{0}$ and $V_{1}$ can be easily given explicit analytical expressions. In order to compute the functions $\Delta(\widetilde{g}), P(\widetilde{g})$, and $V(\widetilde{g})$ at higher $\widetilde{g}$, Eqs. (22) can be solved numerically by increasing $\widetilde{g}$ stepwise through a simple iteration procedure. The obtained dependence of the net dc velocity $V_{\mathrm{dc}}=(V(A)+V(-A)) / 2$ versus the density $n$ of repelling particles is shown in Fig. 5(a). The results are in good qualitative agreement with simulations of the Langevin equations ${ }^{1}$ with a pair potential

$$
W(y)=g \frac{\lambda-|y|}{\lambda^{2}} \text { if }|y|<\lambda \text { and } W=0 \text { otherwise. }
$$

Figure 5(a) (solid magenta and open blue circles) clearly shows that, as predicted in our theoretical analysis within the effective potential approach, the net current for repelling particles increases (decreases) with density at high (low) barriers $Q$ with respect to the driving $A$. The comparison between numerics and theory reveals a quantitative disagreement [circles versus dashed curves in Fig. 5(a)]. This apparent discrepancy points to unavoidable corrections to our meanfield scheme, including an appreciable screening of the interparticle interaction, and nonlocality effects introduced by the truncated pair potential $W$ used in the simulation. To make the approximate analytical curves reproduce closer the corresponding simulation data, the bare interaction constant $g$ $=g_{\mathrm{MD}}$ employed in the simulation must be replaced in Eqs. (22) by a rescaled interaction constant $g_{\mathrm{MF}}$, namely $g_{\mathrm{MF}}$ $\simeq(2 / 3) g_{\mathrm{MD}}$ [solid curves versus circles in Fig. 5(a)].

For the case of attractive particles, the solution of Eq. (22) has been obtained up to a certain critical point, i.e., for $n<n_{\text {crit }}$. This indicates the condensation phase-transition, which is now driven by the force amplitude $A$. Numerical simulations qualitatively agree with analytical ones: The ratchet current increases with the density $n$ of attractive particles up to the condensation point $n_{\text {crit }}$ [Figs. 5(b) and 5(c) (red squares)]. For densities above the condensation threshold $n_{\text {crit }}$, different scenarios can take place. If the amplitude $A$ of the ac force is smaller than both substrate restoring forces $Q / l_{1}$ and $Q / l_{2}$, then the particles condense in the tilted potential wells, no matter what the orientation of the drive. Since the mobility of the condensed particles is zero, the average net particle current drops to zero [Fig. 5(b)]. Most notably, if $A$ takes values between the two substrate forces $Q / l_{1}, Q / l_{2}$, i.e., $f_{1}<A<f_{2}$, then potential wells exist only in one tilting configuration (here, $U+A x$ ). Therefore, in our simulation the particles condense at the minima of $U+A x$ when the ac force pushes them to the left; the instantaneous current in such "hard" direction drops to zero. On the contrary, the particles are almost ballistic when the periodic force pushes them in the opposite, "easy" direction (no minima and therefore no condensation in $U-A x$ ). The stroboscopic spatial distribution of attracting particles subject to an ac drive pointing in the hard and the easy direction, respectively, are shown in the inset of Fig. 5(c): When pushed in the hard direction, almost all particles condense at the bottom of the wells; on the contrary, particles moving to the right in the running state are distributed quite homogeneously in space. Therefore, motion is allowed in the "easy" or natural ratchet direction only; the curve $V_{\mathrm{dc}}(n)$ levels off in correspondence with the condensation density $n_{\text {crit }}$, i.e., it saturates for $n>n_{\text {crit }}$ [Fig. 5(c), red squares]. For larger ac forces with $A>\max \left(f_{1}, f_{2}\right)$, no condensation occurs in either direction and $V_{\mathrm{dc}}(n)$ monotonically approaches a saturation value [inset in Fig. 5(b)] which decreases with increasing $A$.

\section{SPONTANEOUS SYMMETRY BREAKING}

In order to understand more precisely what occurs at the condensation transition, we study the particle distributions for both equilibrium and nonequilibrium cases.

We plot the particle distributions $F_{1}$ below and above the condensation point (Fig. 6). Simulating two substrate potential cells, we found that both cells are equivalently occupied at low particle densities (see point 1, below the condensation point). Above condensation (points 2 and 3), the spatial equivalence of the two cells is spontaneously broken: Par- 


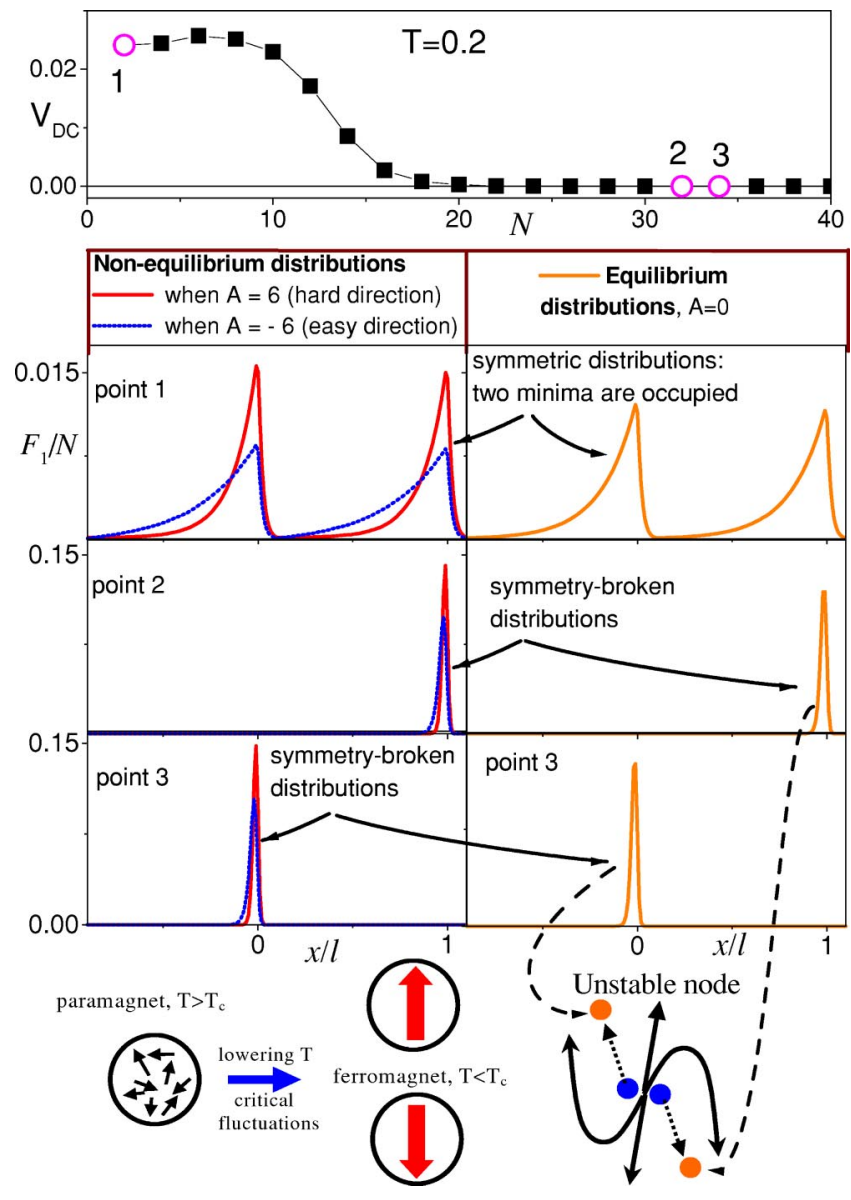

FIG. 6. Spontaneous symmetry-breaking destroys the equivalence of two neighboring potential cells at the phase transition. The normalized particle distributions $F_{1}(x / l) / N$ are shown for three different total numbers $N=2 n$ of particles inside the two cells, corresponding to the net velocity $V_{\mathrm{dc}}(N)$ shown at the top. The normalized equilibrium distributions $F_{1}(x / l) / N$ for the same particle numbers are also shown in the right column. These results were obtained by numerically solving the Langevin equations (1). The parameters used here are $\nu=0.01, Q=1, l_{1}=0.9, \lambda=0.1, g=-0.02, T=0.2, A$ $=0.5$. Both cells are equally occupied at densities lower than the condensation point (e.g., point 1); this equivalence is broken at higher densities (points 2 and 3). Bottom sketches: The mechanism of spontaneous symmetry breaking at the condensation point has counterparts in the theory of equilibrium phase transitions (e.g., the paramagnetic-ferromagnetic transition shown in the left sketch at the bottom and certain dynamical instabilities of the type sketched in the right sketch at the bottom, as described in the text).

ticles condense either on the right or the left minimum, no matter if the initial particle distribution was set the same in both cells. This is the manifestation of very small fluctuations getting strongly amplified in time. Note that the translational symmetry of the substrate may be broken for both nonequilibrium (at relatively weak driving) and equilibrium operating conditions (no external drive), alike. This is different from the essentially nonequilibrium symmetry-breaking phase transition reported in Ref. 27, that disappears in equilibrium and requires two noise sources. For zero substrate potential, the condensation transition is replaced by the transition from weakly coupled to clustered particle motion discussed in the next section.

The symmetry-breaking mechanism discussed here consists in the irregular accumulation of particles in some sub- strate wells, with the remaining wells getting completely depleted. This occurs via the following amplification of fluctuations: very minor, random differences in particle occupation of different cells become more and more pronounced as time evolves and finally break the original equivalence of all cells. This is somewhat similar to the "Maxwell demon" mechanism where particles originally equally distributed among cells accumulate in some wells while depleting other wells. Also, it provides a deep analogy between this nonequilibrium dynamics near the condensation point and dynamical instabilities or critical-fluctuations near symmetry-breaking phase transitions (see, e.g., Ref. 37). For instance, critical fluctuations at the critical temperature produce a symmetry-broken ferromagnetic state (with either up or down magnetization along a ferromagnetic easy-axis) from a fully symmetric paramagnetic phase (Fig. 6, left bottom sketch). By analogy, if two particles start their motion from an unstable equilibrium position, they can move far apart from one another depending on very minor differences in their initial conditions (Fig. 6, right bottom sketch). Note that such an analogy between equilibrium phase transitions and instability of dynamical systems has been successfully used in the renormalization group approach.

\section{BINARY MIXTURE: CONTROLLING MOTION OF PASSIVE PARTICLES}

In this section we consider how to control the motion of particles in binary mixtures. We consider transport in quasione-dimensional geometries, thus including the wide category of fabricated devices and nano-biological systems addressed in recent literature..$^{7,11-13,34,35}$ Since the dragging effect implies "trapping" the target species $B$ by another species $A$, we first need to take into account the local change in the distribution of $B$ particles near an $A$ particle. This can be done by considering the binary distribution function $F_{A B}\left(x, x^{\prime}\right)$, which describes the probability of finding an $A$ particle near $x$ and a $B$ particle near $x^{\prime}$. A partial differential equation for $F_{A B}$ can be constructed by averaging the time derivative of the microscopic binary distribution

$$
N_{A B}=\sum_{i, j} \delta\left(x-x_{A, i}(t)\right) \delta\left(x^{\prime}-x_{B, j}(t)\right),
$$

over different stochastic realizations. Here, the sum has to be taken over the coordinates $x_{A, i}$ and $x_{B, j}$ of all the $A$ and $B$ particles at time $t$. As our main goal is to study the behavior of one species relative to the other, we further neglect the interaction among particles of the same type.

The relevant Langevin equations are

$$
\frac{d x_{a, i}}{d t}=-\frac{\partial}{\partial x_{a, i}}\left[U_{a}+\sum_{j, j^{\prime}} W\left(x_{A, j}-x_{B, j^{\prime}}\right)\right]+\sqrt{2 k_{B} T} \xi_{a}^{(i)},
$$

where $\xi_{a}^{(i)}$ are white noises with $\left\langle\xi_{a}^{(i)}\right\rangle=0,\left\langle\xi_{a}^{(i)}(t) \xi_{b}^{(j)}(0)\right\rangle$ $=\delta_{a, b} \delta_{i, j} \delta(t)$, and $a, b=A$ or $B$, and $W\left(x_{A, j}-x_{B, j^{\prime}}\right)$ denotes the interaction between the $j$ th $A$ particle and the $j^{\prime}$ th $B$ particle. We assume that the $A$ species is driven by the timedependent force $f_{A}(t)$, possibly in the presence of a periodic asymmetric substrate Eq. (19), while the $B$ species is not 
subject to an asymmetric substrate but only to the external force $f_{B}(t)$; namely $U_{A}=U-f_{A}(t) x$, and $U_{B}=-f_{B}(t) x$. The Langevin equations can be manipulated to determine the time evolution of $F_{A B}$ at $B$ densities $n_{B}$ much lower than the $A$ density $n_{A}$ :

$$
\begin{aligned}
\frac{\partial F_{A B}}{\partial t}= & \frac{\partial}{\partial x}\left\{F_{A B} \frac{\partial\left[U_{A}(x)+W\left(x-x^{\prime}\right)\right]}{\partial x}+k_{B} T \frac{\partial F_{A B}}{\partial x}\right\} \\
& +\frac{\partial}{\partial x^{\prime}}\left\{F_{A B} \frac{\partial\left[U_{B}\left(x^{\prime}\right)+W\left(x-x^{\prime}\right)\right]}{\partial x^{\prime}}\right. \\
& \left.+\int d x^{\prime \prime} F_{A B A}\left(x, x^{\prime}, x^{\prime \prime}\right) \frac{\partial W\left(x^{\prime}-x^{\prime \prime}\right)}{\partial x^{\prime}}+k_{B} T \frac{\partial F_{A B}}{\partial x^{\prime}}\right\},
\end{aligned}
$$

where $F_{A B A}\left(x, x^{\prime}, x^{\prime \prime}\right)$ is the three-particle distribution function related to the probability of finding two $A$ particles near $x$ and $x^{\prime \prime}$ and a $B$ particle near $x^{\prime}$.

Next, we express the three-particle distribution $F_{A B A}$ in terms of one-particle $F_{a}$ and binary distribution $F_{A B}$ functions. In general, a binary function can be written as

$$
F_{A B}\left(x, x^{\prime}\right)=F_{A}(x) F_{B}\left(x^{\prime}\right) G\left(\frac{x+x^{\prime}}{2}, x-x^{\prime}\right)
$$

where $F_{A}$ and $F_{B}$ are the one-particle distributions for $A$ and $B$ and $G$ defines the deviation of the distribution of the $A$ particles near a $B$ particle. In other words, the product $F_{A} G$ determines the conditional probability to find an $A$ particle at $x$ given that a $B$ particle is at $x^{\prime}$. Since we assume zero $A-A$ interactions, the conditional probability to find one $A$ particle at $x$ is approximately independent on the conditional probability to find another $A$ particle at $x^{\prime \prime}$ (or at least these correlations are relatively weak). Thus, the three-particle distribution can be expressed as

$$
\begin{aligned}
F_{A B A}\left(x, x^{\prime}, x^{\prime \prime}\right)= & F_{B}\left(x^{\prime}\right) F_{A}(x) \\
& \times G\left(\frac{x+x^{\prime}}{2}, x-x^{\prime}\right) F_{A}\left(x^{\prime \prime}\right) \\
& \times G\left(\frac{x^{\prime}+x^{\prime \prime}}{2}, x^{\prime \prime}-x^{\prime}\right) .
\end{aligned}
$$

To make the problem analytically tractable we also consider the interaction range $\lambda$ of the $A-B$ interactions to be much smaller than $l$. In such a case we can assume long distances $\left|x-x^{\prime}\right| \gg \lambda$ (where $G\left(\left(x+x^{\prime}\right) / 2, x-x^{\prime}\right)=1$ ) in order to derive $F_{A}$ and $F_{B}$, and short distances $\left|x-x^{\prime}\right| \leqslant \lambda$ to calculate $G$. In the long distance limit, we obtain the Fokker-Planck equations for $F_{A}$ and $F_{B}$ :

$$
\begin{aligned}
& F_{A} \partial_{x} U_{A}+k_{B} T \partial_{x} F_{A}=-j_{A}, \\
& F_{B}\left(\partial_{x}\left[U_{B}+g F_{A}\right]+\beta F_{A}\right)+k_{B} T \partial_{x} F_{B}=-j_{B}
\end{aligned}
$$

with $\partial_{x}=\partial / \partial x$, the effective interaction constant

$$
g(x)=\int d y W(y) G(x, y)
$$

and dragging coefficient

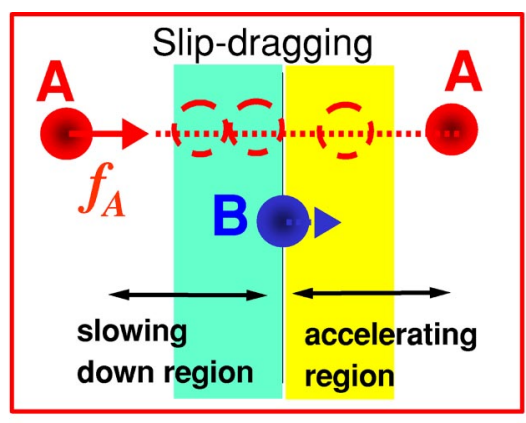

Clustered-dragging $\left(N_{\mathrm{A}}, N_{\mathrm{B}}\right)=(3,1)$ cluster

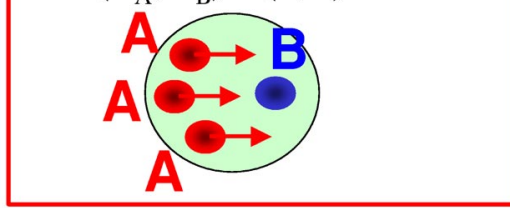

\begin{tabular}{l|l|l|l|l|l|}
\cline { 2 - 6 } & $\left(\mathrm{N}_{\mathrm{A}}, \mathrm{N}_{\mathrm{B}}\right)$ & $(1,1)$ & $(2,2)$ & $(2,1)$ & $(3,1)$ \\
\cline { 2 - 6 } $\begin{array}{l}\text { Clustering- } \\
\text { unclustering } \\
\text { phase transition }\end{array}$ & $\mu$ & $1 / 2$ & $1 / 2$ & $2 / 3$ & $3 / 4$ \\
\cline { 2 - 6 } & $A_{d c}^{\text {crit }}$ & 16 & 32 & 24 & 32 \\
\hline
\end{tabular}

FIG. 7. The upper schematic diagram: The dragging of $B$ (blue particle) by a passing red $A$ particle; the $B$ particle feels a larger density of $A$-particles behind than in front of $B$, due to the slowing down of $A$ approaching $B$ and accelerating $A$ moving away from $B$. The lower schematic picture shows the clustered motion of three $A$ and one $B$ particles. The bottom table shows the effective mobility and critical driving amplitude for cluster destruction.

$$
\beta(x)=\int d y W(y) \frac{\partial G(x, y)}{\partial y}-\frac{1}{2} \frac{\partial g(x)}{\partial x} .
$$

The particle currents $j_{a}$ are defined as $\partial F_{a} / \partial t=-\partial j_{a} / \partial x$ and, in the adiabatic approximation studied below, depend on the instantaneous value of the driving forces $f_{a}(t)$. The equation for the correcting factor $G(x, y)$ to the mean-field (MF) approximation with $y=x-x^{\prime}$ can be easily constructed by imposing $|y| \lesssim \lambda \ll l$ in Eq. (25). For the sake of simplicity, here we only display the case when $U=0$, i.e.,

$$
\frac{\partial}{\partial y}\left\{G\left(\frac{\partial W(y)}{\partial y}-V_{A B}\right)+k_{B} T \frac{\partial G}{\partial y}\right\}=0,
$$

where

$$
V_{A B} \equiv V_{A}-V_{B}=\frac{j_{A}}{n_{A}}-\frac{j_{B}}{n_{B}}
$$

is the relative velocity. Therefore, the $A-B$ interaction produces: (1) An effective potential $g(x) F_{A}$ acting on the $B$ particles, which were originally insensitive to the substrate, and (2) an effective drag $\beta(x) F_{A}$ exerted by the $A$ s on the $B$ s.

\section{A. Dragging and time asymmetric driving: Controlling particle motion without a substrate}

\section{1. dc-Dragging by auxiliary particles}

When no force acts on the $B$ species, the dc-driven $A$ particles can drag along the $B$ particles. When $U=0$ [Eq. 

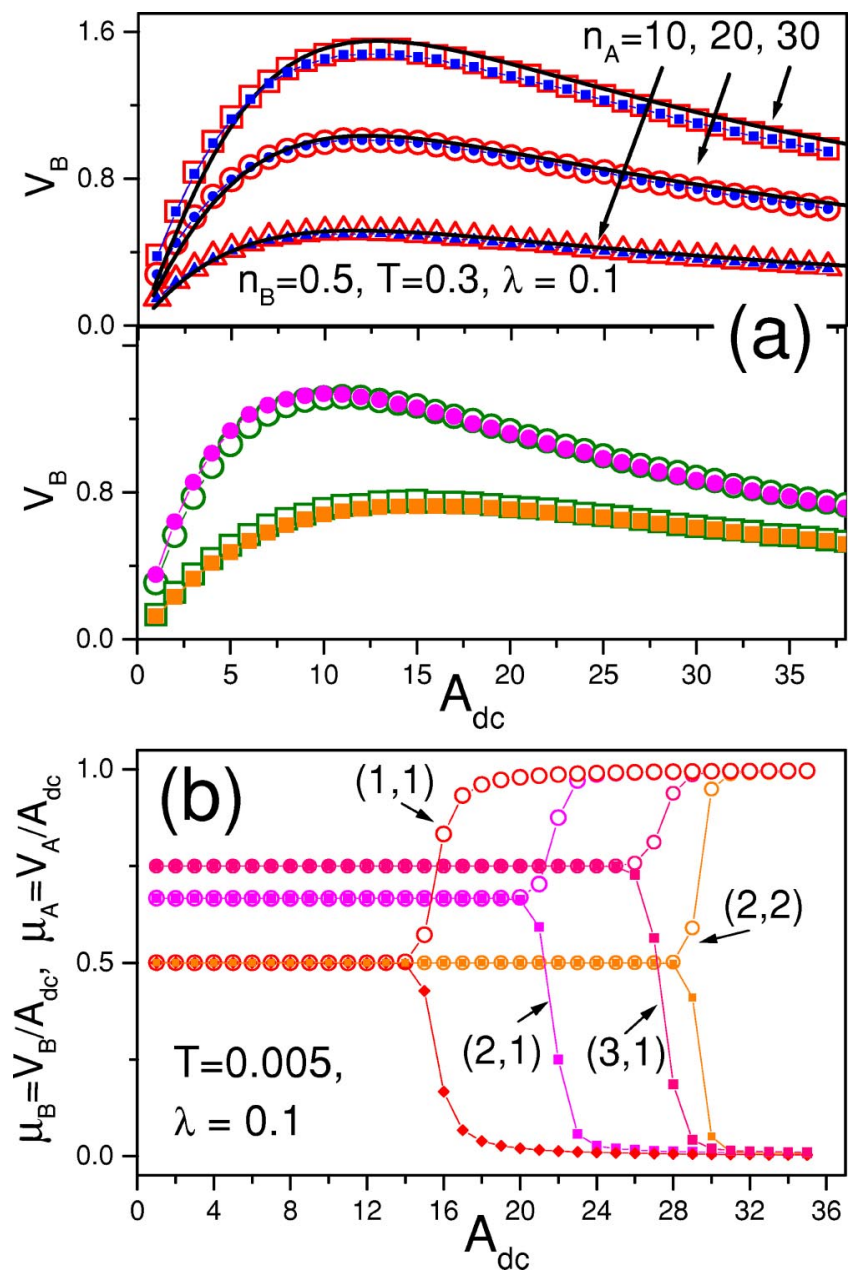

FIG. 8. Dragging particles $B$ by auxiliary particles $A$ in the case of $n o$ substrate, $U=0$, and interaction strength $g= \pm 0.02$. [(a), upper panel] Symbols are from MD simulations (red open and blue solid symbols for repulsive and attractive $A-B$ interactions, respectively) with time step $d t$ $=0.00047$; black lines are the results of analytical calculations. [a, lower panel] The green open (magenta solid) circles and olive open (orange solid) squares are data for $n_{A}=40, g=+0.02(g=-0.02)$ and nonzero attractive (repulsive) interactions between the same particles with $g_{A}=g_{B}=-0.005\left(g_{A}=g_{B}=-0.01\right)$. (b) The mobility of $A$ and $B$ particles vs dc force $A_{\mathrm{dc}}$ obtained from the MD simulations for lower temperature, and repulsive interactions. The different numbers of particles in a cluster are chosen as $\left(N_{A}, N_{B}\right)=(1,1)$ red, $(2,2)$ orange, $(2,1)$ magenta, $(3,1)$ pink.

(31)], the dragging problem (with $f_{B}=0$ and $f_{A}=A_{\mathrm{dc}}$ ) is solved analytically. If $j_{A}=j_{B}=0$, the function $G$ is a simple Boltzmann distribution $G=\exp \left(-W / k_{B} T\right)$, while if $V_{A}>0$ one obtains

$$
G=\frac{V_{A B}}{2 k_{B} T} \int_{y}^{\infty} d z \exp \left\{\frac{W(z)-W(y)-V_{A B}(z-y) / 2}{k_{B} T}\right\} .
$$

Inserting $G$ in Eq. (28) yields $V_{A}=f_{A}$,

$$
V_{B}=n_{A} \int d y G(y) \frac{\partial W}{\partial y} .
$$

In order to understand the physical picture of this dragging effect, let us consider a driven particle $A$ (red particle in the upper "slip-dragging" sketch in Fig. 7) approaching a
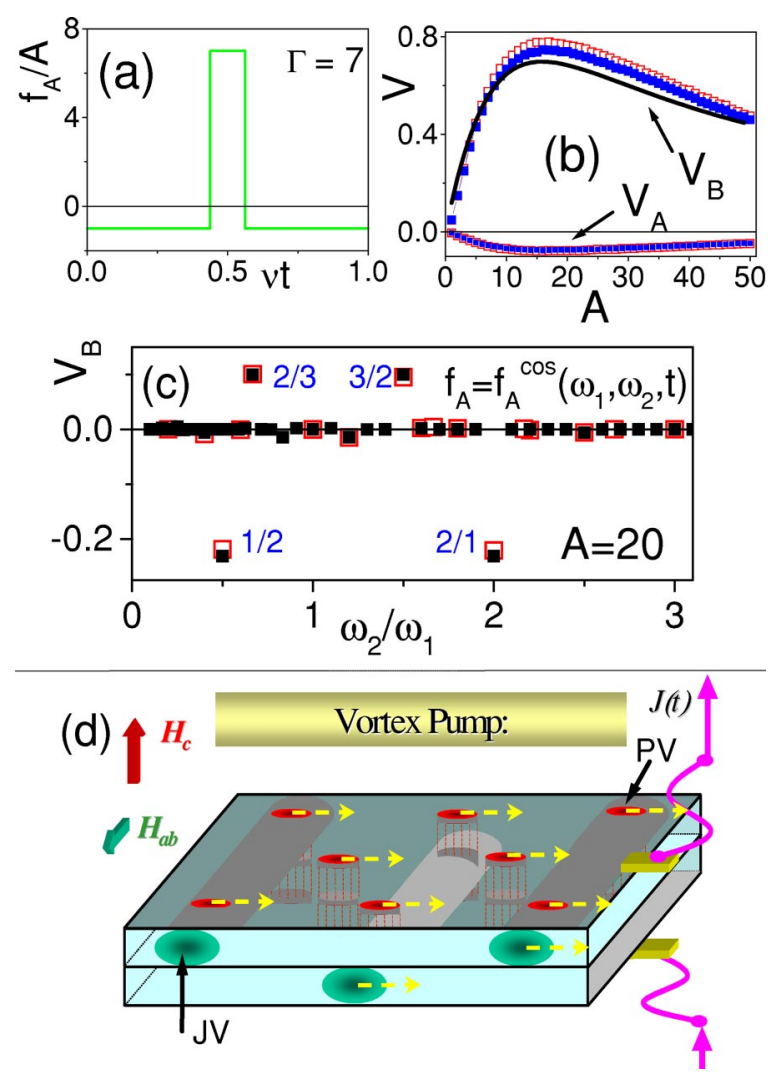

FIG. 9. The net velocities $V_{A}$ and $V_{B}$, from numerically solving Langevin equation (24), vs driving amplitude $A$ (panel b) or frequencies $\omega_{2} / \omega_{1}$ (panel c) for $\lambda=0.075$. The time-asymmetric signal used in (b) is shown in (panel a). Red open and blue solid symbols in (b) correspond to repulsive and attractive interactions, respectively. The black line in (b) and black solid squares in (c) represent $V_{B}$ calculated analytically. (d) Vortex pump uses the time asymmetric electrical current $J(t)$ pushing Josephson vortices (JVs), shown in green, back and forth. Due to the attraction between JVs and pancake vortex stacks (PVs), shown in red, this time asymmetric drive results in dc motion of both JVs and PVs. The densities of JVs and PVs can be easily controlled by an externally applied magnetic field having both inplane $H_{a b}$ and out-of-plane $H_{c}$ components.

nondriven particle $B$ (blue particle). For repelling $A-B$ interactions, the $A$ particle is slowing down when it is behind the $B$ particle (i.e., in the green "slowing down" region). As soon as the $A$ particle overtakes the $B$ particle (i.e., $A$ occurs in the yellow "accelerating" region in front of $B$ in the upper sketch in Fig. 7), the $B$ particle pushes $A$ forward, accelerating the motion of $A$. Because of the faster motion of $A$ in front of $B$ (the yellow region in the upper sketch of Fig. 7), compared to when $A$ is behind $B$ (the green region in the same sketch), the $A$ particle spends more time (or can be found with higher probability) behind $B$ than in front of $B$. This results in an effective force acting on $B$, i.e., pushing $B$ in the direction of the $A$ particle motion.

Next, we performed numerical simulations of the Langevin equations for the $A$ and $B$ particles with interactions described in (23). In spite of the finite interaction length $\lambda$ introduced in our simulation, the analytical equation for $V_{B}$ obtained above describes fairly closely our data in the upper panel of Fig. 8(a), showing that the dragging effect attains a maximum for a certain value of $A_{\mathrm{dc}}$. Introducing the pair interaction between the particles of the same type $\left(W_{a}(y)\right.$ 

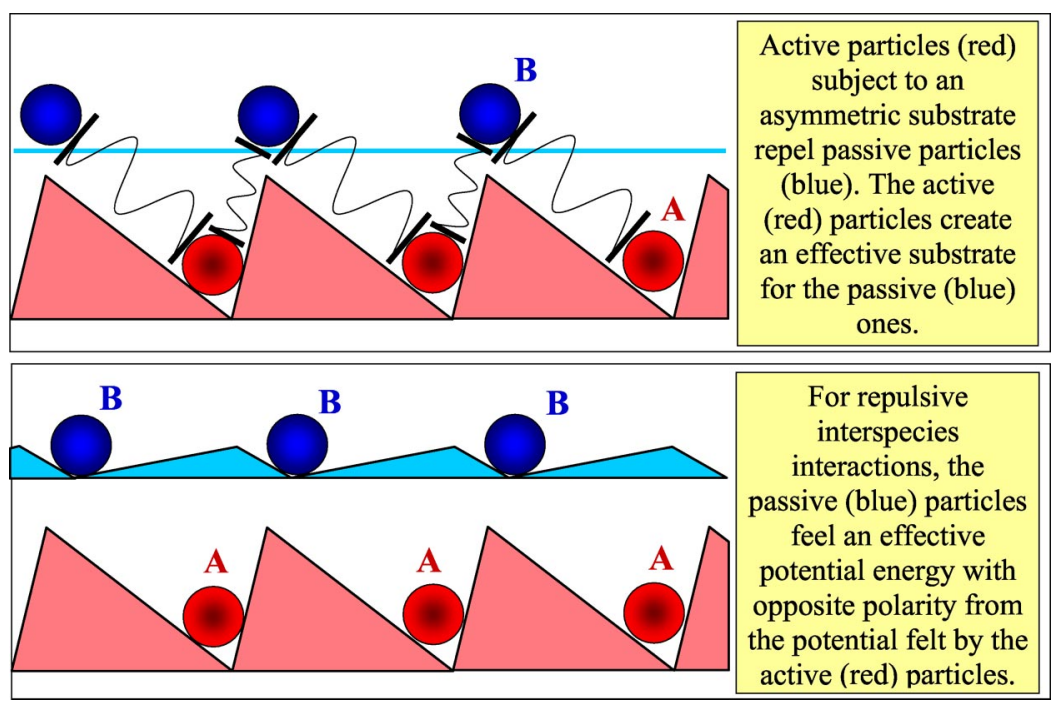

FIG. 10. Schematic diagram showing the mechanism of the mediated ratchet effect: The active $A$ particles (shown in red) occupy the potential minima and repel passive (insensitive to the substrate) $B$ particles (shown in blue) to the maxima of the substrate for the case of repulsive $A-B$ interactions. This can be interpreted as the creation of an effective substrate for $B$ particles with opposite polarity with respect to the substrate felt by the $A$ particles. $=g_{a}(\lambda-|y|) / \lambda^{2}$ if $|y|<\lambda$ and $W_{a}=0$ otherwise), we found a similar dependence of $V_{B}$ on $A_{\mathrm{dc}}$ [see the lower panel in Fig. $8(\mathrm{a})]$ also for the case when interactions between particles of the same type (i.e., $A-A$ and $B-B$ interactions) are taken into account. With decreasing temperature, the solution of the derived transcendental equation for $V_{B}$ vanishes, signaling the occurrence of a dynamical phase transition. Indeed, for weak driving forces, all the $A$ and $B$ particles tend to cluster together (see the lower schematic diagram in Fig. 7 for a cluster having three driven (red $A$ ) particles and one (blue $B$ ) nondriven particle). In order to estimate both the maximum driving force $A_{\mathrm{dc}}^{\text {crit }}$ for the clusters to be stable and their translational velocity $V_{\text {clust }}$, we introduce force-balance equations for clustered $N_{A}$ particles of type $A$, and $N_{B}$ particles of type $B$ at $T=0$ :

$$
V_{A}=V_{\text {clust }}=f_{A}-N_{B} f_{\text {int }} ; \quad V_{B}=V_{\text {clust }}=N_{A} f_{\text {int }}
$$

with interaction force $f_{\text {int }} \leqslant \max \left|\partial_{y} W\right|=|g| / \lambda^{2}$. Thus, we obtain

$$
V_{\text {clust }}=\frac{N_{A} f_{A}}{N_{A}+N_{B}}
$$

for a dc driving force $A_{\mathrm{dc}}<A_{\mathrm{dc}}^{\text {crit }}$ and

$$
A_{d c}^{\text {crit }}=\left(N_{A}+N_{B}\right) \cdot \max \left|\partial_{y} W\right| .
$$

This gives the cluster mobility $\mu_{\text {clust }}=V_{\text {clust }} / A_{\mathrm{dc}}$ $=1 / 2,1 / 2,2 / 3,3 / 4$ and critical force $A_{\mathrm{dc}}^{\text {crit }}(|g|=0.02, \lambda$ $=0.05)=16,32,24,32$ for clusters with $\left(N_{A}, N_{B}\right)$ $=(1,1),(2,2),(2,1),(3,1)$, respectively (see the table in Fig. $7)$. These numbers are in good agreement with the simulation results of Fig. 8(b).

\section{Rectifying the ac dragging}

The dragging effect may be used to induce a net motion of both $A$ and $B$ particles in the absence of a substrate, $U$ =0: As an additional ingredient, a time asymmetric zeroaverage force, like sinusoidal forces

$$
f_{A}(t)=f_{A}^{\cos }\left(\omega_{1}, \omega_{2}\right)=A\left(\cos \omega_{1} t+\cos \omega_{2} t\right)
$$

with $\omega_{2}=2 \omega_{1}{ }^{36}$ or the rectangular waveform of Fig. 9(a), must be applied to one species, say the auxiliary particles $A$. Indeed, applying the alternate signals $f_{A}=-\Gamma A$ and $f_{A}=A$, during the time intervals $t_{1}=1 /(1+\Gamma) \nu$ and $t_{2}=\Gamma /(1+\Gamma) \nu$, respectively, forces time-periodic particle flows with frequency $\nu$. The net $B$ current can be written as

$$
\left\langle V_{B}\right\rangle_{t}=\frac{V_{B}\left(f_{A}=\Gamma A\right)+\Gamma V_{B}\left(f_{A}=-A\right)}{1+\Gamma}
$$

with time-asymmetry factor $\Gamma$. The average $\left\langle V_{B}\right\rangle_{t}$ can be easily calculated through our analytical expression for the thermally averaged $V_{B}$ as well as from simulations; data and analytical results compare very well [Fig. 9(b)]. The rectification due to the $A-B$ dragging can also be seen as spikes or resonances [Fig. 9(c)] on the dependence of the net velocities $V_{B}$ and $V_{A}$ on the frequency $\omega_{2}$, if the signal $f_{A}$ $=f_{A}^{\cos }\left(\omega_{1}, \omega_{2}\right)$ with two-frequencies is applied. When changing $\omega_{2} / \omega_{1}$, the change of the sign of the net velocities allows to effectively control the motion of both species.

\section{B. Mediated ratchet effect}

If the $A$ particles move on an asymmetric substrate, the equation for $G$ becomes complicated. Thus, we will now consider a mean-field (MF) approximation when $G=1 .^{31,32,34,35}$ Even though dragging is lost in such an approximation $(\beta=0)$, the effective potential acting upon the target $B$ particles can be qualitatively reproduced.

When only one species of particles (say, $A$ ) feels the substrate potential (see Fig. 10), active particles $A$ accumulate in the potential wells and repel passive particles $B$ away from these locations towards the maximum of the substrate potential. This results in distributions of $A$ and $B$ particles having different asymmetry [Fig. 11(a)] for repulsive $A-B$ interactions. In contrast, for attractive $A-B$ interactions, both particles accumulate in the substrate minima as shown in our simulations [see Fig. 11(b)]. This can be described using the effective potential approach discussed above for the one spe- 


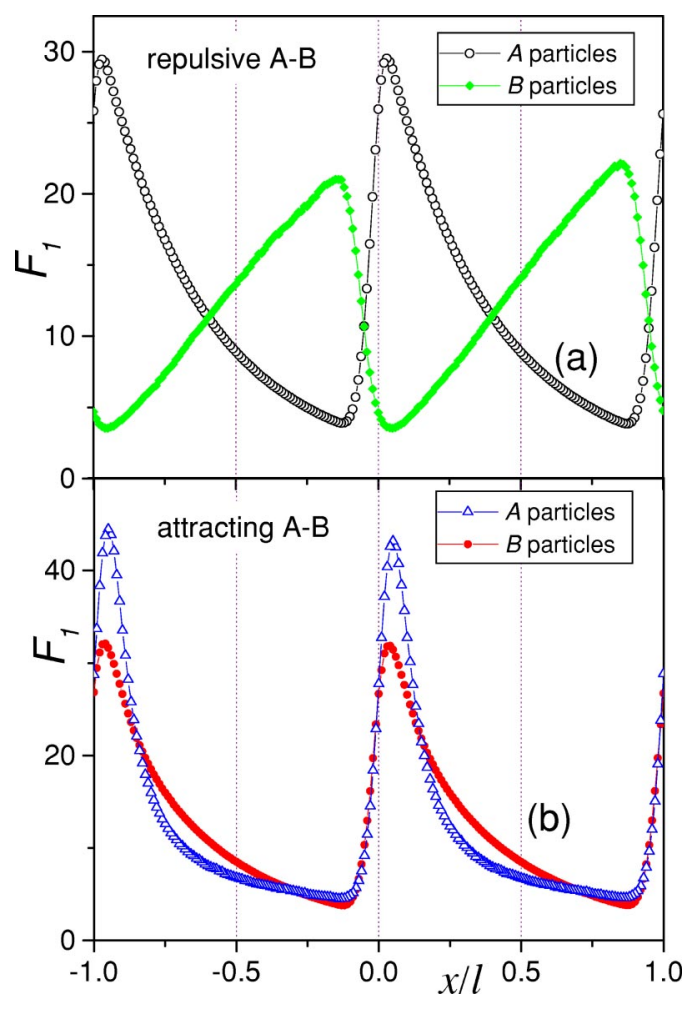

FIG. 11. Distribution of active A and passive B particles for the repulsive (a) or attractive (b) interspecies interactions. The other parameters are: $Q=1$, $l_{1}=0.9, n_{A}=200, n_{B}=12, \lambda=0.05$.

cies case. Namely, using a perturbation approach to Eq. (28), we derive the effective potentials and temperatures for moving fraction of particles as

$$
U_{a}^{\mathrm{eff}}=U_{a}+g_{a A} \phi_{A}^{(0)}+g_{a B} \phi_{B}^{(0)}, \quad k_{B} T_{a}^{\mathrm{eff}}=k_{B} T+g_{a a} \phi_{a}^{(0)},
$$

where $\phi_{a}^{(0)}$ are equilibrium distributions. The renormalization of the diffusion constant (or effective temperature) and the potential experienced by the fraction $\psi_{a}$ of moving particles accounts for their interaction with the remaining particles of both species. Thus, the effective potentials have the opposite polarity for repulsive $A-B$ species [Fig. 12(a)] and the same polarity for attractive $A-B$ species [Fig. 12(b)].

\section{Gating effect: Flashing effective potential}

In the adiabatic approximation ${ }^{1}$ Eqs. (28) yield for the net current of the $a$-species

$$
J_{a}=\nu \int_{0}^{1 / \nu} d t j_{a}\left(f_{A}(t), f_{B}(t)\right),
$$

with

$$
j_{a}=n_{a} l k_{B} T \frac{1-\exp \left(-l f_{a} / k_{B} T\right)}{\int_{0}^{l} d x \int_{x}^{x+l} d z \exp \left(\left[U_{a}^{*}(z)-U_{a}^{*}(x)\right] / k_{B} T\right)},
$$

where $U_{A}^{*}=U_{A}$ and

$$
U_{B}^{*}\left(x, f_{A}\right)=g_{\mathrm{MF}} F_{A}\left(x, f_{A}\right)-f_{B} x,
$$

with $g_{\mathrm{MF}}=\int d y W(y)$. The polarity (or asymmetry) of $g_{\mathrm{MF}} F_{A}$ coincides with the polarity of the original substrate $U$ for attractive interaction, $g_{\mathrm{MF}}<0$, and vice versa for repulsive $A-B$ potentials, $g_{\mathrm{MF}}>0$. Therefore, the ac motion of $B$ particles can be rectified on this potential ("mediated" ratchet effect), i.e., the $B$ particles, which do not originally feel any substrate, perceive a spatial asymmetry due to the $A-B$ interaction. Thus, when the motion of $B$ particles is governed by a "mediated" ratchet effect, one expects the $A$ and $B$ net currents flowing in the same directions if $g_{\mathrm{MF}}<0$, or in the opposite direction if $g_{\mathrm{MF}}>0$. However, there is an additional effect controlling the $B$ motion as the effective potential $g_{\mathrm{MF}} F_{A}$ changes with time. When the force $f_{A}(t)$ points against the steeper substrate slopes (the "hard-motion direction"), the $A$ particles tend to accumulate near the $U$ minima.

\section{a) Repelling A-B species}

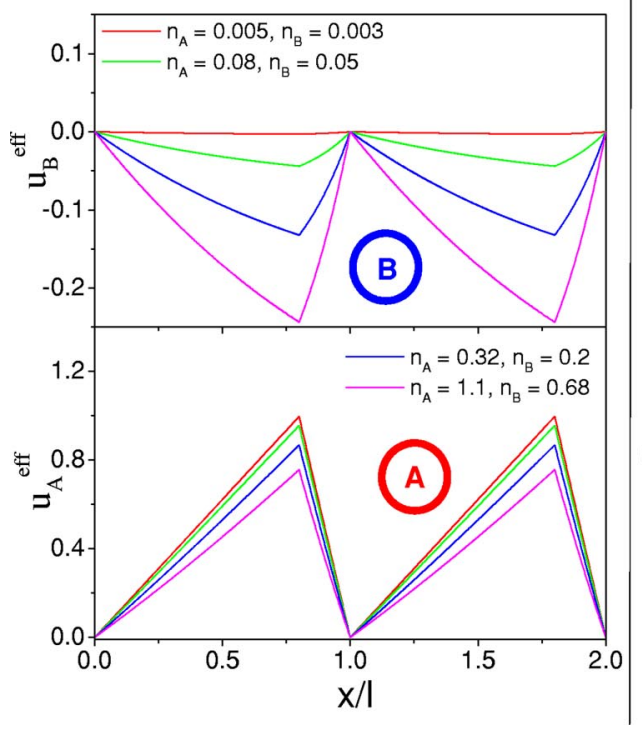

\section{b) Attracting A-B species}

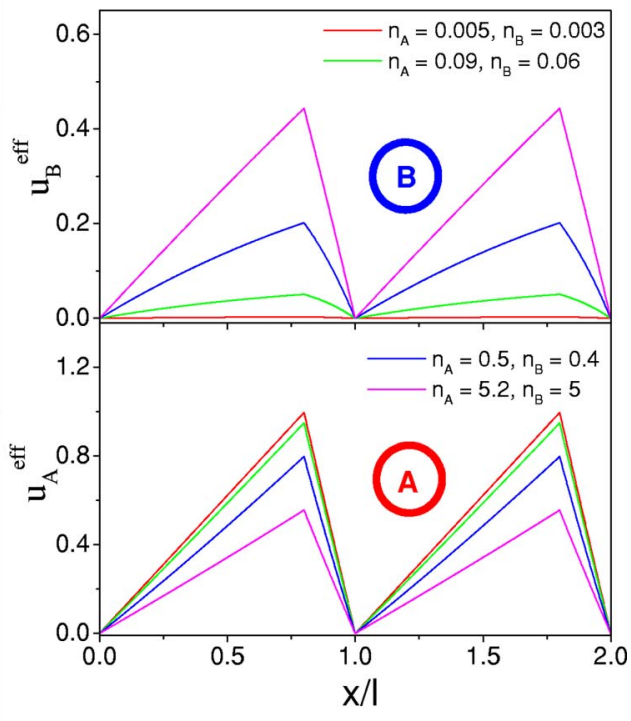

FIG. 12. Spatial dependence of the effective potentials $U_{A}^{\text {eff }}, U_{B}^{\text {eff }}$ at different densities of the $A$ and $B$ particles. In both panels, particles of the same type repel one another; the interaction between particles of different species is repulsive in (a) and attractive in (b). There is no "bare" potential for the $B$ particles, $U_{B} \equiv 0$, whereas the ratchet potential $U_{A}(x)$ is piece-wise linear (19) and here $Q=1, l_{1}=0.8$. The other coupling parameters are: $g_{A A}=g_{B B}$ $=\left|g_{A B}\right|=1$ and $k_{B} T=1$. 

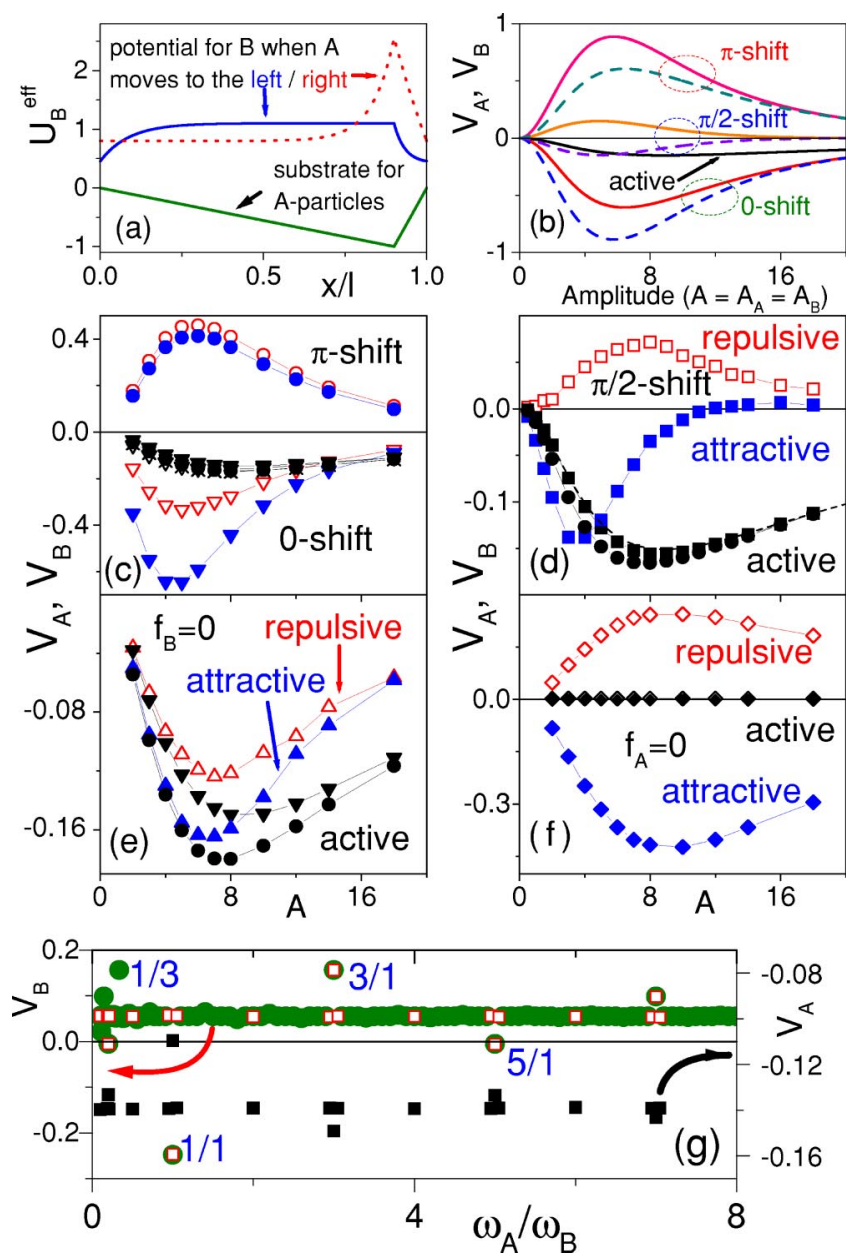

FIG. 13. How to control the net velocities $V_{A}, V_{B}$ by ac forces $f_{A}$ and $f_{B}$ on an asymmetric substrate potential [green profile in (a)] coupled to the $A$ particles, only. We set $\lambda=0.075, n_{A}=50, n_{B}=1, d t=0.00047,|g|=0.02, Q$ $=-1, l_{1}=0.9$. (a) The effective MF potential (red dashed and blue solid landscapes) felt by the $B$ particles when the $A$ particles are forced towards the "hard" (to the right) or to the "easy" (to the left) directions, respectively. (b) $V_{A}$ and $V_{B}$ vs the ac amplitude $A$ of $f_{A}$ and $f_{B}$ calculated in the MF approximation Eqs. (41), (42) for the repulsive (attractive) $A-B$ interaction and in-phase (red solid and blue dashed), out-of-phase (pink solid and light blue dashed) and $\pi / 2$-shifted (orange solid and violet dashed) ac forces. (c) The MD data of $V_{B}$ for repulsive (attractive) species and in-phase (red open (blue solid) triangles) and opposite-phase (red open (blue solid) circles) driving forces; black symbols mark $V_{A}$. (d) The same as in (c) with red open (blue solid) squares for repulsive (attractive) interactions and $\pi / 2$-shifted ac forces, black symbols are $V_{A}$. (e) The ac force is applied only to the $A$ species (i.e., $\left.f_{B}=0\right) . V_{B}$ is marked by red open (blue solid) up-triangles for repulsive (attractive) interactions, the corresponding $V_{A}$ marked by downtriangles (circles). (f) The ac force is applied only to $B$ particles $\left(f_{A}=0\right), V_{A}$ is very weak (black symbols), but the ac motion of $B$ particles is rectified by an effective asymmetric potential $\left(V_{B}\right.$ is plotted by red open (blue solid) symbols for repulsive (attractive) interactions). (g) The dependence of the net velocities $V_{A}$ (black squares from MD) and $V_{B}$ (red open symbols from MD and green filled circles calculated analytically and linearly scaled to fit MD data) on the frequency ratio $\omega_{B} / \omega_{A}$ (odd ratios provide peaks) for repulsive interactions and $A_{A}=A_{B}=8, \phi_{A}=\phi_{B}=0$.

Thus, due to the repulsive (attractive) $A-B$ interactions, this strongly nonuniform distribution of $A$ particles causes high peaks (deep wells) in the effective potential acting on the $B$ particles [Fig. 13(a)]. The ensuing high potential barriers of $U_{B}^{\text {eff }}$ significantly slow down the $B$ particle motion (gating effect) when the $A$ particles move in their "hard" direction. In contrast, the relatively faster motion of the $A$ particles as
$f_{A}(t)$ pushes them in the opposite, "easy" direction, corresponds to shallower $U_{B}^{\text {eff }}$ barriers [blue solid line in Fig. 13(a)] and, thus, to a higher $B$ mobility. The time-correlation of these stopping intervals for $B$ particles (when the $A s$ move in the "hard" direction) and ac-driving $f_{B}(t)$ of $B s$ results in another way to control the motion of $B s$.

\section{Interplay between gating, mediated-ratchet, and dragging effects in a rocked ratchet}

Let us consider ac drives of the form

$$
f_{A}(t)=A_{A} \operatorname{sgn}\left[\cos \left(\omega_{\mathrm{A}} \mathrm{t}+\phi_{\mathrm{A}}\right)\right]
$$

and

$$
f_{B}(t)=A_{B} \operatorname{sgn}\left[\cos \left(\omega_{\mathrm{B}} \mathrm{t}+\phi_{\mathrm{B}}\right)\right] .
$$

If the frequencies and amplitudes of both signals coincide $\omega_{A}=\omega_{B}=\omega, A_{A}=A_{B}=A$, we can restrict the discussion to three main cases depending on the relative phase of the ac forces: (i) In-phase drives: $\phi_{A}=\phi_{B}$; (ii) opposite-phase drives: $\phi_{A}=\phi_{B}+\pi$; and (iii) $\pi / 2$-shifted drives: $\phi_{A}=\phi_{B}$ $+\pi / 2$. In the first two cases the gating effect is dominant and the direction of the $B$ current does not depend on the polarity of $g_{\mathrm{MF}} F_{A}$, i.e., the sign of $V_{B}$ is insensitive to the sign of the $A-B$ interactions (attractive or repulsive). Indeed, the $A$ particles, when pushed against the steeper slopes of $U$, create the high barriers of $U_{B}^{\text {eff }}$ [Fig. 13(a)] that lock the motion of $B$ particles as long as $f_{B}$ pushes them to the right or to the left in the case of in-phase or opposite-phase ac drives. Thus, the $A$ and $B$ particles drift necessarily to the same or opposite direction for cases (i) or (ii), respectively. In contrast, when $f_{A}(t)$ and $f_{B}(t)$ are phase shifted by $\pi / 2$, the $B$ particle motion is governed by the asymmetry of the effective potential $g_{\mathrm{MF}} F_{A}$. During the half ac cycle when the effective potential $U_{B}^{\text {eff }}$ develops high (low) barriers, the $B$ particles are being pushed directly by $f_{B}(t)$ to the right and to the left for the same amount of time. Thus, the $B$ particles are driven back and forth on the asymmetric ratchet potentials $g_{\mathrm{MF}} F_{A}\left(x, f_{A}\right.$ $=A)$ and $g_{\mathrm{MF}} F_{A}\left(x, f_{A}=-A\right)$, alternately. Since the polarity of these potentials (due to "mediated" ratchet effect) depends on the sign of the interaction $g_{\mathrm{MF}}$, attractive $A$ and $B$ particles move together $\left[\operatorname{sgn}\left(J_{A}\right)=\operatorname{sgn}\left(J_{B}\right)\right]$, while repulsive particles travel in opposite directions $\left[\operatorname{sgn}\left(\mathrm{J}_{\mathrm{A}}\right)=-\operatorname{sgn}\left(\mathrm{J}_{\mathrm{B}}\right)\right]$.

Examples of MF calculations for in-phase, oppositephase and $\pi / 2$-shifted drives are shown in Fig. 13(b). Our numerics prove that dragging effects may correct the MF estimates of $V_{B}$, so as to break the symmetry with respect to the interaction sign [see Fig. 13(c) for cases (i) and (ii) and Fig. 13(d) for (iii)]. Nevertheless, the main MF picture remains valid. In order to clearly separate dragging and rectification effects, we performed simulations with $A_{A} \neq 0, A_{B}$ $=0$ [Fig. 2(e)] and with $A_{A}=0, A_{B} \neq 0$. For the first case (dragging), the $A$ and $B$ particles drift in the same direction, while in the second case (mediated ratchet) the sign of $V_{B}$ is determined by the sign of the $A-B$ interactions. Finally, if we fix amplitudes and phases, for instance, $A_{A}=A_{B}$ and $\phi_{A}$ $=\phi_{B}$, and change the frequency ratio $\omega_{A} / \omega_{B}$, we obtain velocity spikes for commensurate values of $\omega_{A}$ and $\omega_{B}$. Indeed, in the incommensurate case the gating effect is irrelevant and 

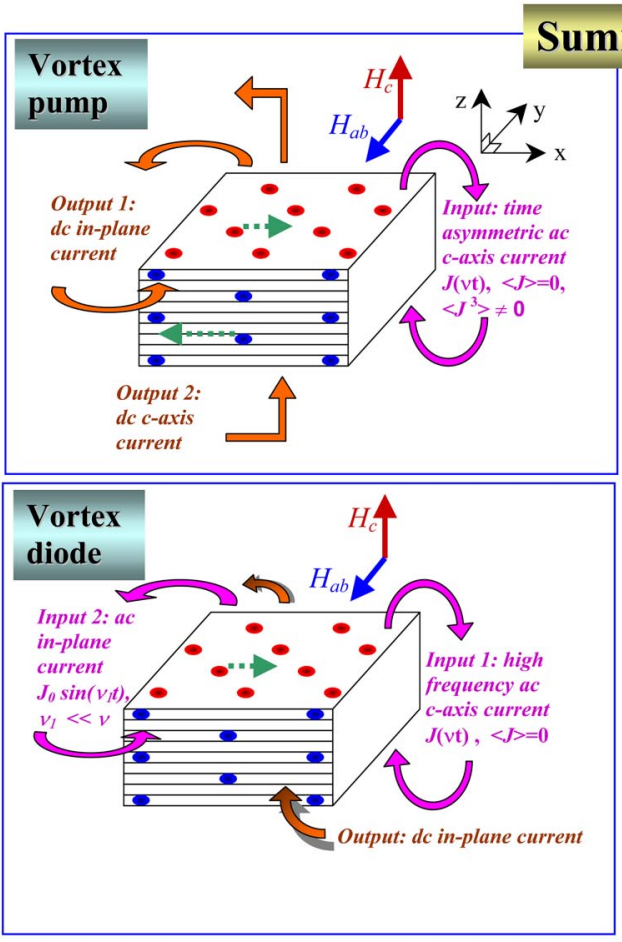

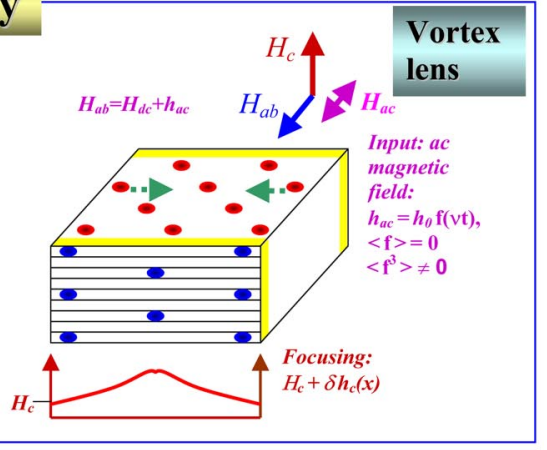

(a) The vortex pump converts the time-asymmetric oscillations of the JV's, induced by the timeasymmetric applied ac c-axis current, into a de drift of both PV's and JV's.

(b) The vortex diode uses the spatially asymmetric ratchet potential generated by the oscillating JV's, which are driven by a high-frequency c-axis current, to rectify the applied ac current $J_{\mathrm{ab}}$ $=J_{0} \sin v_{1} \mathrm{t}$ flowing along the in-plane magnetic field.

(c) The vortex lens utilizes an applied timeasymmetric ac magnetic field to increase or decrease the PV density at the center of the sample.
FIG. 14. Three different devices (Ref. 11) use time-asymmetric drives to rectify motion of two vortex species: Josephson vortices and pancake vortex stacks. the net motion is determined by a combination of mediated ratchet and dragging effect. However, if the frequencies of the driving signals are commensurate, the modulation of the effective potential $U_{B}^{\text {eff }}$ gets time-correlated with the direct ac drive $f_{B}(t)$, thus resulting in large deviations of $V_{B}$ from its incommensurate baseline [see Fig. $13(\mathrm{~g})$ ]. Note that the velocity spikes ${ }^{38}$ shown in Fig. 13(g) result from spatial asymmetries (i.e., they disappear if $U=0$ ); in contrast, the velocity spikes [see Fig. 9(c)] for commensurate values of $\omega_{1}$ and $\omega_{2}$ in the absence of a substrate, are caused by the residual nonlinearity of the symmetric problem $V_{B}\left(f_{A}\right)=-V_{B}\left(-f_{A}\right)$. [As a result, in Fig. 9(c) spikes happen at different winding numbers with respect to Fig. 13(g).]

\section{CONTROLLING VORTEX MOTION USING EITHER TIME-ASYMMETRIC DRIVE OR SPATIAL- ASYMMETRIC SUBSTRATE}

As one of the possible experimental realizations of our proposal, let us now consider an interesting vortex system ${ }^{39}$ in layered superconductors like Bi2212 placed in an external oblique magnetic field (Fig. 14). The $a b$-field component $H_{a b}$ generates Josephson vortices (JVs) trapped between superconducting layers [green vortices in Fig. 9(d)]. JVs are usually very weakly pinned and can be driven by the electrical current $J_{c}(t)$ flowing along the $c$ axis. This results in the rocked potential

$$
U_{\mathrm{JV}}=\Phi_{0} J_{c}(t) x / c,
$$

with flux quanta $\Phi_{0}$ and light speed $c$. Note, that this problem can be reduced to one-dimensional (1D) because of the translational invariance along $H_{a b}$. The $c$ axis magnetic field component generates pancake vortex stacks (PVs) [red vortices in Fig. 9(d)], which can be easily driven by an in-plane ac current $J_{a b}(t)$ and subject to a periodic pinning potential
$U_{\text {pin }}$ fabricated by using irradiation or lithography. This results in the rocking potential for PVs:

$$
U_{\mathrm{PV}}=\Phi_{0} J_{a b}(t) x / c+U_{\mathrm{pin}} .
$$

The mutual interaction $W_{\mathrm{PV}-\mathrm{JV}}$ between PVs and JVs is the short-range attraction interaction. ${ }^{11}$ The parameters of vortex interactions and pinning can be easily controlled by temperature and magnetic fields. Since vortex dynamics is described by overdamped equations, the problem of the mutual PV-JV transport is reduced to the one considered in this paper. Thus, the binary mixture of PVs and JVs is an experimentally accessible system where the effects discussed above can be observed. For instance, several devices which drag PVs by $\mathrm{JV}_{\mathrm{s}}$ using time-asymmetric drives have been discussed ${ }^{11}$ (see Fig. 14). Recent experiments ${ }^{13,14}$ confirm our theoretical predictions. $^{11}$

Another vortex system exhibiting features of binary mixtures was studied on a superconducting/magnetic hybrid with magnetic triangles and a superconducting film. ${ }^{12}$ The vortices, pinned by magnetic traps and jumping from one triangle to another, play the role of an active species for such system, while the interstitial vortices (moving outside traps) can be considered as a passive species (see Fig. 15). The mediated ratchet effect produces the rectification in such a superconducting/magnetic hybrid. This allows us to interpret $^{12}$ the observed current inversion.

\section{CONCLUSIONS}

The stochastic transport of interacting particles, for single species and binary mixtures, is described both analytically (using Fokker-Planck-type equations for many-particle distribution functions) and numerically (simulating Langevin equations). The rectification power of one-species ratchet devices can be tuned by changing the particle density and 

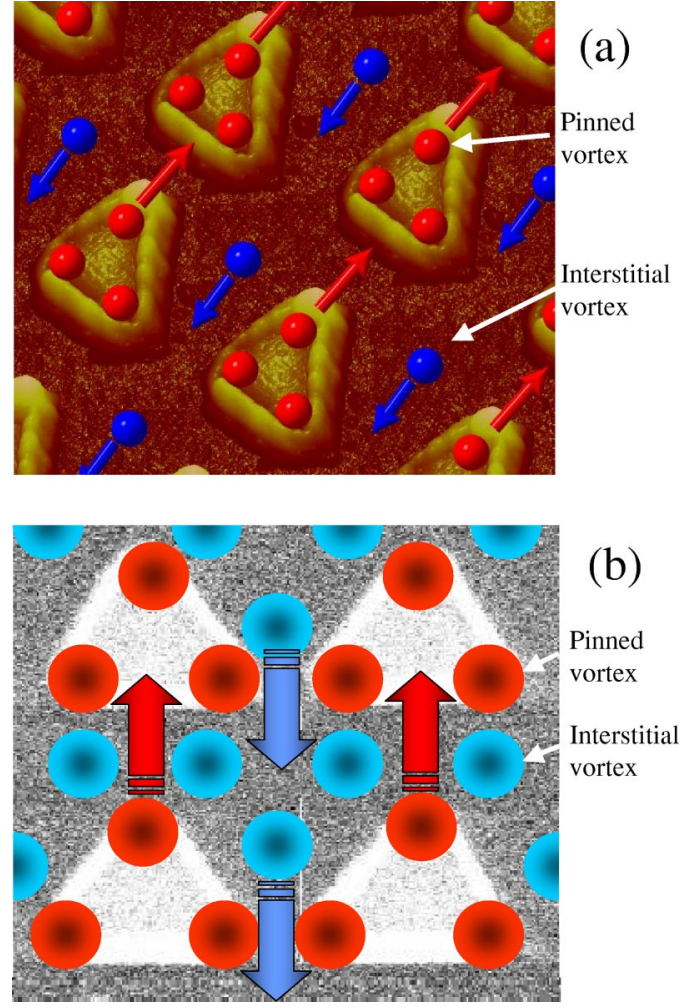

FIG. 15. Sketch of the position of the vortices for several matching fields (Ref. 12). Vortices pinned on the triangles are shown in red and interstitial vortices in blue. Red and blue arrows show the direction of the net flow of these vortices due to the mediated ratchet effect. Three-dimensional (3D) (a) and two-dimensional (2D) (b) sketches correspond to the fourth and sixth matching fields, respectively.

described using effective potentials accounting for both substrate and particle interactions. Moreover, a huge enhancement of the rectification can be obtained for attracting particles near and above the condensation transition when a macroscopic amount of particles pile up or condense in the substrate minima. We describe ways to control the motion of passive (insensitive to substrate and/or driving forces) species in binary mixtures, including dc and ac dragging passive species by active ones, mediated ratchet effect, and gating (flashing) mechanisms for guiding both components of the binary mixture.

We study here the net motion of interacting particles, while the other interesting issue-their diffusion-calls for further investigation. Note that even the diffusion of a single particle in a washboard potential exhibits very intriguing properties, ${ }^{40}$ which become more complex for interacting particles.

\section{ACKNOWLEDGMENTS}

Most of the work reviewed here has been done in collaboration with Professor Fabio Marchesoni. We are very grateful to him for his contributions.

This work was supported in part by the National Security Agency (NSA) and Advanced Research and Development Activity (ARDA) under Air Force Office of Research
(AFOSR) Contract No. F49620-02-1-0334; and also supported by the U.S. National Science Foundation Grant No. EIA-0130383.

${ }^{1}$ See, e.g., the reviews: P. Reimann, Phys. Rep. 361, 57 (2002); R. D. Astumian and P. Hänggi, Phys. Today 55, 33 (2002); F. Jülicher, A. Ajdari, and J. Prost, Rev. Mod. Phys. 69, 1269 (1997); R. D. Astumian, Science 276, 917 (1997); J. M. R. Parrondo and B. J. De Cisneros, Appl. Phys. A: Mater. Sci. Process. 75, 179 (2002); P. Hänggi, F. Marchesoni, and F. Nori, Ann. Phys. 14, 51 (2005).

${ }^{2}$ P. Reimann, R. Bartussek, R. Haussler, and P. Hänggi, Phys. Lett. A 215 , 26 (1996).

${ }^{3}$ R. Bartussek, P. Hänggi, and J. P. Kissner, Europhys. Lett. 28, 459 (1994); M. O. Magnasco, Phys. Rev. Lett. 71, 1477 (1993).

${ }^{4}$ A. L. R. Bug and B. J. Berne, Phys. Rev. Lett. 59, 948 (1987); J. Prost, J. F. Chauwin, L. Peliti, and A. Ajdari, Phys. Rev. Lett. 72, 2652 (1994).

${ }^{5}$ D. A. Doyle, J. M. Cabral, R. A. Pfuetzner, A. Kuo, J. M. Gulbis, S. L. Cohen, B. T. Chait, and R. MacKinnon, Science 280, 69 (1998).

${ }^{6}$ K. Kitamura, M. Tokunaga, A. H. Iwane, and T. Yanagida, Nature (London) 397, 129 (1999).

${ }^{7}$ For reviews see: H. Linke, Appl. Phys. A: Mater. Sci. Process. 75, 167 (2002); special issue on Ratchets and Brownian Motors: Basics, Experiments and Applications; P. Hänggi, M. Ratner, and S. Yaliraki, Chem. Phys. 281, 111 (2002); issue on Transport in Molecular Wires.

${ }^{8}$ J. Rousselet, L. Salome, A. Ajdari, and J. Prost, Nature (London) 370, 446 (1994).

${ }^{9}$ I. Derényi, C. Lee, and A. L. Barabási, Phys. Rev. Lett. 80, 1473 (1998).

${ }^{10}$ J. F. Wambaugh, C. Reichhardt, C. J. Olson, F. Marchesoni, and Franco Nori, Phys. Rev. Lett. 83, 5106 (1999); C.-S. Lee, B. Jankó, I. Derényi, and A.-L. Barabási, Nature (London) 400, 337 (1999); C. J. Olson, C. Reichhardt, B. Jankó, and F. Nori, Phys. Rev. Lett. 87, 177002 (2001); F. Marchesoni, B. Y. Zhu, and F. Nori, Physica A 325, 78 (2003); B. Y. Zhu, F. Marchesoni, and F. Nori, Physica E (Amsterdam) 18, 318 (2003); 18, 322 (2003); Phys. Rev. Lett. 92, 180602 (2004); B. Y. Zhu, F. Marchesoni, V. V. Moshchalkov, and F. Nori, Phys. Rev. B 68, 014514 (2003); Physica C 388, 665 (2003); 404, 260 (2004).

${ }^{11}$ S. Savel'ev and F. Nori, Nat. Mater. 1, 179 (2002).

${ }^{12}$ J. E. Villegas, S. Savel'ev, F. Nori, E. M. Gonzalez, J. V. Anguita, R. García, and J. L. Vicent, Science 302, 1188 (2003).

${ }^{13}$ D. Cole, A. Crisan, S. J. Bending, T. Tamegai, K. van der Beek, and M. Konczykowski, Physica C 404, 99 (2004).

${ }^{14} \mathrm{~S}$. Bending et al., (unpublished).

${ }^{15}$ Y. Togawa et al., (unpublished); J.Van de Vondel et al.., Phys. Rev. Lett. 94, 057003 (2005).

${ }^{16}$ S. Matthias and F. Muller, Nature (London) 424, 53 (2003); C. Kettner, P. Reimann, P. Hänggi, and F. Müller, Phys. Rev. E 61, 312 (2000).

${ }^{17}$ Z. Siwy and A. Fuliński, Phys. Rev. Lett. 89, 198103 (2002); C. Marquet, A. Buguin, L. Talini, and P. Silberzan, Phys. Rev. Lett. 88, 168301 (2002).

${ }^{18}$ P. T. Korda, M. B. Taylor, and D. G. Grier, Phys. Rev. Lett. 89, 128301 (2002); B. A. Koss and D. G. Grier, Appl. Phys. Lett. 82, 3985 (2003).

${ }^{19}$ D. G. Grier, Nature (London) 424, 810 (2003).

${ }^{20}$ A. Gopinathan and D. G. Grier, Phys. Rev. Lett. 92, 130602 (2004).

${ }^{21}$ K. Ladavac and D. G. Grier, Opt. Express 12, 1144 (2004).

${ }^{22}$ S. Lee, K. Ladavac, M. Polin, and D. G. Grier, Phys. Rev. Lett. 94, 110601 (2005).

${ }^{23}$ F. Marchesoni, Phys. Rev. Lett. 77, 2364 (1996).

${ }^{24}$ I. Derényi and T. Vicsek, Phys. Rev. Lett. 75, 374 (1995).

${ }^{25}$ J. Buceta, J. M. Parrondo, C. Van den Broeck, and F. J. de la Rubia, Phys. Rev. E 61, 6287 (2000).

${ }^{26}$ C. Van den Broeck, I. Bena, P. Reimann, and J. Lehmann, Ann. Phys. 9, 713 (2000).

${ }^{27}$ P. Reimann, R. Kawai, C. Van den Broeck, and P. Hänggi, Europhys. Lett. 45, 545 (1999).

${ }^{28}$ R. Eichhorn, P. Reimann, and P. Hänggi, Phys. Rev. Lett. 88, 190601 (2002).

${ }^{29}$ C. Van den Broeck, J. M. R. Parrondo, and R. Toral, Phys. Rev. Lett. 73, 3395 (1994)

${ }^{30}$ S. I. Denisov, E. S. Denisova, and P. Hänggi, Phys. Rev. E 71, 016104 (2005).

${ }^{31}$ S. Savel'ev, F. Marchesoni, and F. Nori, Phys. Rev. E 70, 061107 (2004).

${ }^{32}$ S. Savel'ev, F. Marchesoni, and F. Nori, Phys. Rev. E 71, 011107 (2005).

${ }^{33}$ J. H. Morais-Cabral, Y. Zhou, and R. MacKinnon, Nature (London) 414, 37 (2001). 
${ }^{34}$ S. Savel'ev, F. Marchesoni, and F. Nori, Phys. Rev. Lett. 91, 010601 (2003).

${ }^{35}$ S. Savel'ev, F. Marchesoni, and F. Nori, Phys. Rev. Lett. 92, 160602 (2004).

${ }^{36}$ F. Marchesoni, Phys. Lett. A 119, 221 (1986).

${ }^{37}$ S. Savel'ev and F. Nori, Phys. Rev. B 70, 214415 (2004).

${ }^{38}$ S. Savel'ev, F. Marchesoni, P. Hänggi, and F. Nori, Europhys. Lett. 67,

179 (2004); Eur. Phys. J. B 40, 403 (2004); Phys. Rev. E 70, 066109
(2004).

${ }^{39}$ A. Tonomura, H. Kasai, O. Kamimura, T. Matsuda, K. Harada, T. Yoshida, T. Akashi, J. Shimoyama, K. Kishio, T. Hanaguri, K. Kitazawa, T. Masui, S. Tajima, N. Koshizuka, P. L. Gammel, D. Bishop, M. Sasase, and S. Okayasu, Phys. Rev. Lett. 88, 237001 (2002); A. Grigorenko, S. Bending, T. Tamegai, S. Ooi, and M. Henini, Nature (London) 414, 728-731 (2001).

${ }^{40} \mathrm{M}$. Borromeo and F. Marchesoni, Chaos (to be published). 